ARTICLE

https://doi.org/10.1038/s41467-019-09375-8

\title{
Oncogenic potential of truncated RXR $\alpha$ during colitis-associated colorectal tumorigenesis by promoting IL-6-STAT3 signaling
}

Xiaohong Ye $e^{1}$, Hua Wur,2,3, Luoyan Sheng ${ }^{1}$, Yi-xin Liu ${ }^{1}$, Fang Ye $e^{1}$, Mo Wang ${ }^{1}$, Hu Zhou ${ }^{1}$, Ying Su ${ }^{3} \&$ Xiao-kun Zhang ${ }^{1,3}$

Retinoid $X$ receptor-alpha $(R X R \alpha)$ is a potent regulator of inflammatory responses; however, its therapeutic potential for inflammatory cancer remains to be explored. We previously discovered that $R X R \alpha$ is abnormally cleaved in tumor cells and tissues, producing a truncated $\mathrm{RXR} \alpha(\mathrm{tRXR} \alpha)$. Here, we show that transgenic expression of $t R X R \alpha$ in mice accelerates the development of colitis-associated colon cancer (CAC). The tumorigenic effect of $\operatorname{tRXR} \alpha$ is primarily dependent on its expression in myeloid cells, which results in interleukin-6 (IL-6) induction and STAT3 activation. Mechanistic studies reveal an extensive interaction between tRXR $\alpha$ and TRAF6 in the cytoplasm of macrophages, leading to TRAF6 ubiquitination and subsequent activation of the NF- $\mathrm{B}$ inflammatory pathway. K-80003, a tRXR $\alpha$ modulator derived from nonsteroidal anti-inflammatory drug (NSAID) sulindac, suppresses the growth of tRXR $\alpha$-mediated colorectal tumor by inhibiting the NF- KB-IL-6-STAT3 signaling cascade. These results provide new insight into $\operatorname{tRXR} \alpha$ action and identify a promising $t R X R \alpha$ ligand for treating CAC.

\footnotetext{
${ }^{1}$ School of Pharmaceutical Sciences, Fujian Provincial Key Laboratory of Innovative Drug Target Research, Xiamen University, 361102 Xiamen, China. ${ }^{2}$ Department of Pathology, Soochow University, 215123 Suzhou, China. ${ }^{3}$ Sanford Burnham Prebys Medical Discovery Institute, 10901 N. Torrey Pines Road, La Jolla, CA 92037, USA. These authors contributed equally: Xiaohong Ye, Hua Wu. Correspondence and requests for materials should be addressed to X.-k.Z. (email: xzhang@sbpdiscovery.org)
} 
$\mathrm{R}$ etinoid X receptor a (RXRa), a master member of the nuclear receptor superfamily, regulates a broad spectrum of cellular processes under physiological and pathophysiological conditions ${ }^{1-3}$. Targeted disruption of RXRa gene leads to preneoplastic lesions in prostate ${ }^{4}$, alopecia, epidermal interfollicular hyperplasia, keratinocyte hyperproliferation, and aberrant terminal differentiation in the skin ${ }^{5}$ and the development of cervical malignant lesions ${ }^{6}$. Altered expression and modification of RXRa is also implicated in the development of a number of malignancies ${ }^{1,3,7-9}$. RXRa binding to promyelocytic leukemia (PML)/RAR $\alpha$ is absolutely required for the development of acute PML in transgenic mice, revealing its oncogenic potential when acting inappropriately ${ }^{10,11}$. Interestingly, RXRa is proteolytically cleaved in cancer cells ${ }^{12-26}$, implying that aberration in RXRa signaling by limited proteolysis plays a role in cancer development. Consistent with its role in cancer development, RXRa is one of the most important targets for the development of pharmacologic intervention and therapeutic applications ${ }^{1,7,27-30}$. Notably, Targretin ${ }^{\circledR}$ was approved for treating human cutaneous $\mathrm{T}$ cell lymphoma ${ }^{27}$. However, the therapeutic potential of RXRa modulators remains to be explored, which requires our further understanding of its role in tumorigenesis.

Chronic inflammation is an important contributor to increased risk of cancer ${ }^{31,32}$. RXRa and ligands are potent regulators of inflammatory responses. RXRa is highly expressed in all inflammatory cell types ${ }^{33}$. Analysis of macrophage-specific RXRa knockout mice revealed a critical role of RXR $\alpha$ in regulating macrophage functions and inflammatory responses, including the upregulation of chemokine expression and reduction of antiviral responses in myeloid cells ${ }^{33-35}$. RXR $\alpha$ compounds also regulate various inflammatory pathways in different cell types ${ }^{36-40}$. Interestingly, certain anti-inflammatory agents, such as docosahexaenoic acid ${ }^{41}, \mathrm{R}$-etodolac ${ }^{42}$, and sulindac ${ }^{25}$, serve as RXRa ligands, further supporting the role of RXRa in regulating inflammatory responses. However, the underlying mechanisms by which RXRa and ligands act, especially whether and how they mediate and modulate the causal link between inflammation and cancer remain obscure.

$\mathrm{RXRa}$, like other nuclear receptors, consists of three distinct domains: an $\mathrm{N}$-terminal $\mathrm{A} / \mathrm{B}$ region, a DNA-binding domain (DBD), and a C-terminal ligand-binding domain (LBD) $)^{1,2,8}$. The presence of well-conserved DBD in RXR $\alpha$ and other nuclear receptors led to the discovery that members of the nuclear receptor superfamily serve as ligand-dependent nuclear transcription factors ${ }^{2}$. Subsequent studies, however, have revealed that RXR $\alpha$ and other nuclear receptors could also act independently of their DNA binding and transcription function ${ }^{9,43}$. Orphan nuclear receptor Nur77 translocates from the nucleus to the cytoplasm where it acts at mitochondria to promote apoptosis ${ }^{44,45}$ and mitophagy ${ }^{46}$, whereas steroid hormone receptors interact with the p85a subunit of phosphoinositide 3-kinase (PI3K) in the cytoplasm to modulate the PI3K survival pathway ${ }^{47,48}$. RXRa is predominantly nuclear but can migrate to the cytoplasm in response to inflammation ${ }^{23,25,49,50}$. We previously reported that RXR $\alpha$ is proteolytically cleaved in cancer cells, resulting in production of a truncated RXRa $(\operatorname{tRXR} \alpha)$ that lacks a portion of its N-terminal A/B domain ${ }^{25}$. Unlike full-length RXRa, tRXRa is predominantly cytoplasmic in response to inflammatory cytokine tumor necrosis factor- $\alpha$ (TNFa), interacting with the p85a to activate the PI3K/AKT pathway $^{25}$. Thus tRXRa plays a critical role in mediating the survival effect of inflammatory signaling through its nontranscriptional action. The role of tRXRa was further illustrated by our finding that tRXRa activity was inhibited by sulindac, a nonsteroidal anti-inflammatory drug (NSAID) $)^{51}$, and analogs $21,25,52$.
Colorectal cancer is closely associated with chronic inflammation ${ }^{53,54}$, and regular use of NSAIDs lowers the mortality from colorectal cancer ${ }^{55}$. RXRa downregulation ${ }^{38}$ or malfunction of RXRa due to phosphorylation ${ }^{56}$ is associated with the development of colorectal cancer. To establish the tumorigenic effect of tRXRa in the development of inflammation-associated cancer and the therapeutic potential of targeting tRXR $\alpha$-mediated inflammatory signaling pathways, we have generated tRXRa transgenic mice. Here we report our characterization of the tRXR $\alpha$ transgenic mice with respect to its tumorigenic effects in the development of colitis-associated colon cancer (CAC), the underlying molecular mechanism, and the therapeutic significance.

\section{Results}

Tumorigenic effect of tRXRa in mouse colon cancer model. To test the tumorigenic effect of tRXR $\alpha$ in the development of inflammation-associated cancer, we generated a loxP-tRXR $\alpha$ $\left(t R X R \alpha^{f l o x}\right)$ transgenic mouse line that contained loxP-flanked SV40 poly A sequences between CAG promoter and tRXRa cDNA sequences (Supplementary Fig. 1a). The loxP-tRXRa mice were crossed with the CMV-Cre mice expressing Cre recombinase under the control of cytomegalovirus (CMV) promoter to produce transgenic tRXRa mice (Tg-tRXRa) (Supplementary Fig. 1b) that express various levels of tRXRa in all the tissues examined (Supplementary Fig. 1c). $T g-t R X R \alpha$ mice, when compared to control wild-type mice, showed certain aberrant crypts in the colon of $T g$ - $t R X R \alpha$ mice (21-month old; Supplementary Fig. 1d). To address the inflammatory effect of tRXR $\alpha, T g$ - $t R X R \alpha$ and control $t R X R \alpha^{\text {flox }}$ mice were subjected to a single injection with the colonotropic mutagen azoxymethane (AOM) followed by three cycles of treatment with the luminal toxin dextran sodium sulfate (DSS) (Fig. 1a), a standard CAC mouse model ${ }^{57}$. We found that $T g-t R X R \alpha$ mice lost less body weight than control mice upon AOM/DSS treatment (Fig. 1b), suggesting that tRXRa expression is involved in the proliferation and survival of colonic epithelial cells. When tumor load was analyzed, $T g$ - $t R X R \alpha$ mice showed increased tumor size $(1.556 \pm 0.07171 \mathrm{~mm}, N=32)$ as compared to control mice (1.281 $\pm 0.06477 \mathrm{~mm}, N=16)$ (Fig. 1c). The number of macroscopic tumors in $T g-t R X R \alpha$ mice $(3.2 \pm$ 0.4667 tumors per mouse, $N=10$ ) was also increased by two-fold when compared to control animals $(1.6 \pm 0.3399$ tumors per mouse, $N=10$ ) (Fig. 1d). As a result, $T g$ - $t R X R \alpha$ mice exhibited a high frequency of larger tumors than control mice (Fig. 1e). Histological analyses confirmed more aberrant crypt foci and hyper-plastic crypts in $T g$ - $t R X R \alpha$ mice (Fig. 1f). These data suggested that tRXRa may play a role in promoting the development of CAC.

To further characterize the role of $t R X R \alpha, T g$ - $t R X R \alpha$ mice were treated with either DSS or AOM. When treated with three cycles of DSS, we found that 2 of the $10 \mathrm{Tg}$ - $t R X R \alpha$ mice developed at least 1 tumor, while none of 12 control mice had tumor under the same treatment (Fig. 1g; Table 1). Histological examination confirmed the presence of adenomas in the colon of $T g$ - $t R X R \alpha$ mice (Fig. $1 \mathrm{~g}$ ). AOM is a potent carcinogen that causes a high incidence of colon cancer in rodents ${ }^{57,58}$. Although none of the 6 control mice showed tumor 6 months after repetitive intraperitoneal treatment of AOM, Tg-tRXR $\alpha$ mice were much more sensitive to AOM treatment with 2 of the $6 \mathrm{Tg}$ - $t R X R \alpha$ mice developing adenoma tumors (Fig. 1h; Table 1), as revealed by histological examination (Fig. 1h), suggesting that tRXRa is involved in modulating the initial response to AOM. Taken together, these data demonstrate that tRXR $\alpha$ acts at different levels to modulate the development of colorectal tumor. 
a

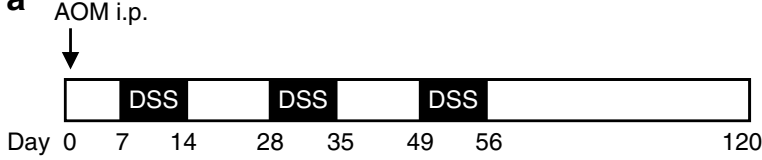

c
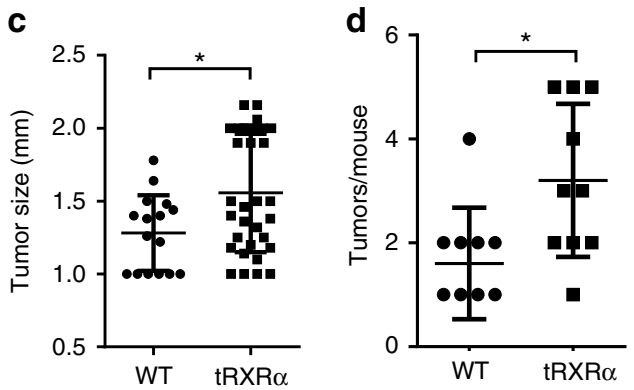

e

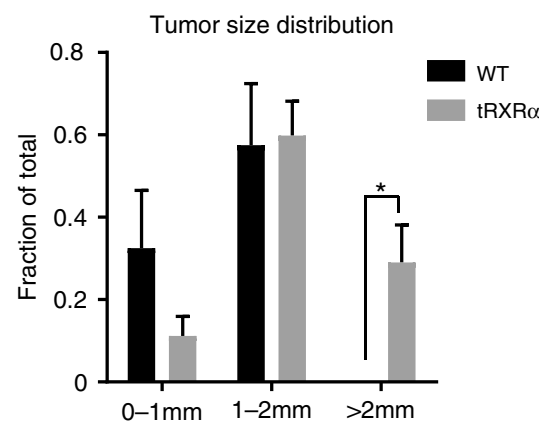

g

\begin{tabular}{|llllll|l|}
\hline DSS & & DSS & & DSS & & \\
\hline & 7 & 21 & 28 & 42 & 49 & 6 months
\end{tabular}

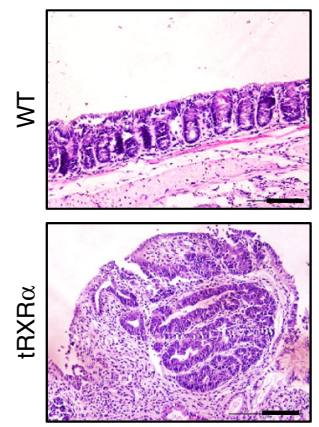

b

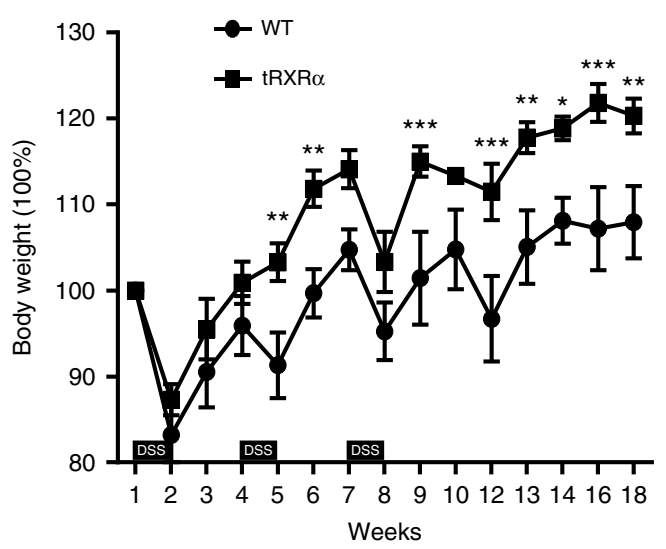

f
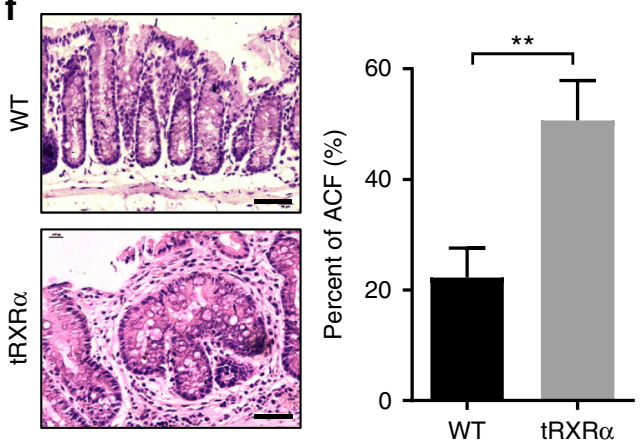

h
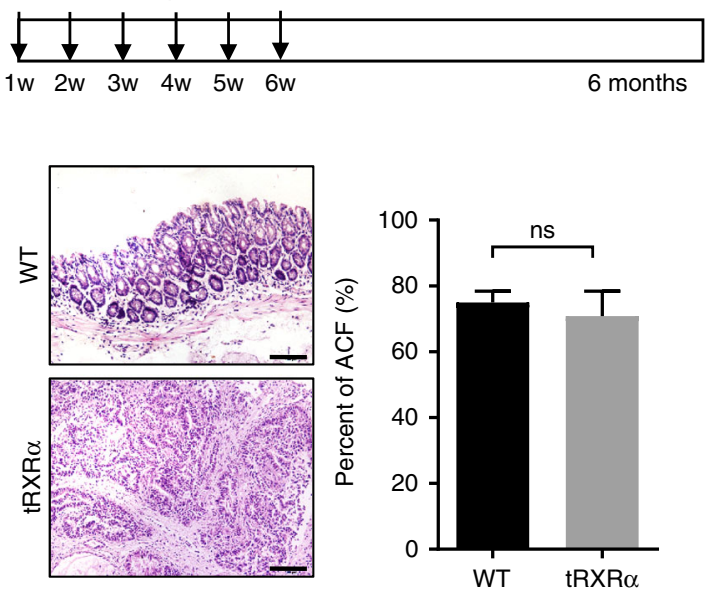

Fig. 1 Tumorigenic effect of truncated retinoid $X$ receptor $\alpha(\operatorname{tRXR} \alpha)$ in colitis-associated colon cancer mouse model. a Schematic representation of the azoxymethane $(A O M) /$ dextran sodium sulfate (DSS) procedure. i.p. intraperitoneal. b Body weight curve of control wild-type (WT) and Tg-tRXR $\alpha$ mice during the course of AOM/DSS colitis. Data are mean \pm SEM (standard error of the mean, $n=7$ ), two-way analysis of variance (ANOVA). c Average tumor size. Each dot represents a tumor. Data are mean \pm SD (standard deviation, $n=10$ ), $t$ test. $\mathbf{d}$ Average tumor number. Each dot represents the tumor number of one individual mouse. Data are mean \pm SD $(n=10), t$ test. e Tumor size distribution. Data are mean \pm SEM $(n=10)$, two-way ANOVA. f Colon tumor sections from mice treated with AOM/DSS at 120 days were microscopically analyzed and examined for microscopic aberrant crypt foci (ACF) after hematoxylin and eosin (H\&E). Scale bars, $50 \mu \mathrm{m}$. Data are mean \pm SEM $(n=10 / 8), t$ test. $\mathbf{g}$ Role of tRXR $\alpha$ in promoting DSS-induced colorectal carcinogenesis. Schematic representation of the 2.5\% DSS procedure (top panel). Colon sections from mice treated with DSS for 6 months were microscopically analyzed and examined for microscopic ACF after H\&E. Scale bars, $100 \mu \mathrm{m}$. Data are mean \pm SEM $(n=10), t$ test. h Role of tRXR $\alpha$ in promoting AOM-induced colorectal carcinogenesis. Schematic representation of the AOM $(10 \mathrm{mg} / \mathrm{kg})$ procedure (top panel). Colon sections from mice treated with AOM for 6 months were microscopically analyzed and examined for microscopic ACF after H\&E. Scale bars, $100 \mu \mathrm{m}$. Data are mean \pm SEM $(n=6)$; ns not significant. ${ }^{\star} P<0.05,{ }^{\star \star} P<0.01,{ }^{\star \star \star} P<0.001$ 
Table 1 Transgenic expression of $\operatorname{tRXR} \alpha$ promotes colorectal carcinogenesis

\begin{tabular}{|c|c|c|c|c|c|c|c|}
\hline \multirow{2}{*}{$\begin{array}{l}\text { Model } \\
\text { Group of mice }\end{array}$} & \multicolumn{4}{|c|}{ DSS alone } & \multicolumn{3}{|c|}{ AOM alone } \\
\hline & \multicolumn{2}{|l|}{ WT } & \multicolumn{2}{|c|}{ tRXR $\alpha$} & \multirow{2}{*}{$\begin{array}{l}\text { WT } \\
6\end{array}$} & \multicolumn{2}{|c|}{ tRXR $\alpha$} \\
\hline Number of mice & 12 & 8 & 1 & 1 & & 4 & 2 \\
\hline Tumors of mouse & 0 & 0 & 2 & 1 & 0 & 0 & 1 \\
\hline Mice with tumours & 0 & & 20 & & 0 & & \\
\hline$P$ value & \multicolumn{4}{|c|}{0.05211} & \multicolumn{3}{|c|}{0.0607} \\
\hline
\end{tabular}

AOM azoxymethane, DSS dextran sodium sulfate, $t R X R \alpha$ trunacted retinoid $X$ receptor $\alpha, W T$ wild type

Activation of STAT3 in colorectal tumors from $T g$ - $t R X R \alpha$ mice. To explore the mechanism by which tRXRa impacts tumorigenesis, we determined the activation of signaling pathways critical for colorectal carcinogenesis in tumor tissues from $T g$ - $R X R \alpha$ mice. In agreement with our previous finding that tRXR $\alpha$ could activate the PI3K/AKT pathway ${ }^{25}$, the level of pAKT was elevated in tumor tissues from $T g$ - $t R X R \alpha$ mice treated with either AOM/DSS (Fig. 2a) or DSS alone (Fig. 2b). The A/B domain of RXR $\alpha$ was shown to interact with $\beta$-catenin to trigger its degradation ${ }^{59}$. Transgenic expression of tRXRa that lacks a large portion of the $\mathrm{A} / \mathrm{B}$ domain resulted in elevated level of $\beta$ catenin independently of the treatment with AOM/DSS (Fig. 2a) or DSS (Fig. 2b), likely due to the dominant-negative effect of tRXRa. Interestingly, glycogen synthase kinase (GSK)-3 $\beta$ phosphorylation at Serine 9, which is a substrate of $\mathrm{AKT}^{60}$, was also elevated in $T g$ - $t R X R \alpha$ mice. As phosphorylation of GSK-3 $\beta$ could inhibit its activity known to induce $\beta$-catenin degradation ${ }^{60}$, enhanced GSK-3 $\beta$ phosphorylation may also play a role in the accumulation of $\beta$-catenin in $T g$ - $t R X R \alpha$ mice. Emerging evidence has now revealed a critical role of signal transducer and activator of transcription factor 3 (STAT3) activation in CAC tumorigenesis ${ }^{61,62}$. We found that the level of phosphorylated STAT3 was consistently elevated in $T g-t R X R \alpha$ mice even in the absence of treatment (Fig. 2a). In agreement with previous reports $^{62}$, AOM/DSS treatment resulted in STAT3 activation in control mice. However, the activation was significantly enhanced in $T g$ - $t R X R \alpha$ mice (Fig. 2a). Tg-tRXR $\alpha$ mice also exhibited higher STAT3 activation upon DSS treatment compared to control mice (Fig. 2b). Enhanced STAT3 activation by tRXRa was confirmed by immunohistochemical staining in tumor tissues from $\mathrm{Tg}$ $t R X R \alpha$ and control mice treated with AOM/DSS or DSS (Fig. 2c). These data revealed for the first time an important role of tRXR $a$ in the activation of STAT3 during colorectal tumorigenesis.

STAT3 could be activated in acute DSS-induced colitis, protecting DSS-induced colonic epithelium injury ${ }^{61,62}$. We next determined whether tRXR $a$ expression impacted the activation of STAT3 in the acute DSS-induced colitis model that uses a single 5 -day course of DSS. Tg-tRXR $\alpha$ and control mice were treated with $3 \%$ DSS in drinking water for 5 days followed by 4 days of recovery in normal drinking water. Starting from day 1 of DSS treatment, $T g-t R X R \alpha$ mice lost significant more weight than control animals but rapidly regained body weight after removal of DSS from the drinking water (Fig. 2d). In contrast, control mice continued to lose weight when DSS was removed from the drinking water for 2 more days. As a result, colon length of $\mathrm{Tg}$ $t R X R \alpha$ mice was significantly longer compared to control mice (Fig. 2e). Consistent with the initial rapid body weight loss, $\mathrm{Tg}$ $t R X R \alpha$ mice showed more epithelial injury and crypt incomplete 2 days after DSS treatment (Supplementary Fig. 2a). When STAT3 activation was assessed, we found that DSS activation of STAT3 occurred much earlier in Tg-tRXR $\alpha$ mice than in control mice. Upregulation of STAT3 phosphorylation was seen 3 days after DSS treatment in control mice. However, enhanced STAT3 activation was clearly seen 1 day after DSS treatment in $T g-t R X R \alpha$ mice and continued to increase after 3- or 5-day treatment (Fig. 2f). The expression of the tissue-protective factors TFF3 and Reglll $\gamma$, the downstream targets of STAT3, which are known to mediate the protective effect of STAT3 on intestinal injury during colitis $^{62}$, was also significantly elevated in $T g$ - $t R X R \alpha$ mice (Fig. 2g). Body weight recovery from DSS treatment is a result of hyperproliferation of epithelial cells caused by STAT3 activation $^{61}$. In line with their rapid recovery from body weight loss, $T g-t R X R \alpha$ mice exhibited less severe epithelial cell damage, loss of crypts, and ulceration compared with control mice after the acute DSS-induced colitis protocol (Supplementary Fig. 2b). Immunohistochemical staining revealed enhanced Ki-67 and proliferating cell nuclear antigen (PCNA) staining in the crypts of $T g-t R X R \alpha$ mice (Supplementary Fig. 2c, d). These results suggested that STAT3 activation might play a role in tRXRainduced protection of intestinal cells from DSS-induced injury and promotion of intestinal epithelial cell survival and proliferation.

Increased infiltration of inflammatory cells in tRXRa tumor. Our finding that STAT3 was strongly activated in Tg-tRXR $\alpha$ tumors prompted us to study the mechanism by which tRXRa promotes STAT3 activation. Cytokines especially interleukin (IL)-6 and IL-11 through their respective receptors can phosphorylate and activate STAT3 ${ }^{61,62}$. Our immunohistochemical study revealed much more pronounced staining of the macrophage marker CD68 in lamina propria of tumor tissue from $\mathrm{Tg}$ $t R X R \alpha$ mice than that from control mice treated with AOM/DSS (Fig. 3a), indicating an increased infiltration of inflammatory cells in $T g-t R X R \alpha$ mice. In tumor developed from $T g-t R X R \alpha$ mice subjected to DSS treatment solely, a significant number of infiltrated inflammatory cells was also detected (Fig. 3b). When the expression levels of cytokines were examined in mice treated with AOM/DSS, we found increased mRNA expression levels of IL-6, IL-11, and TNFa in tumor tissues from $T g$ - $t R X R \alpha$ mice (Fig. 3c). DSS-treated $t R X R \alpha$ mice also showed a noticeable increase in mRNA expression of IL-6 and TNFa but not of IL-11 (Fig. 3d). In contrast, $T g-t R X R \alpha$ mice treated with AOM did not show any apparent effect on macrophage infiltration (Supplementary Fig. 3a) and the expression of IL-6, IL-11, and TNFa, although cyclin D2 expression was increased (Supplementary Fig. 3b). IL-6 produced by lamina propria myeloid cells can protect intestinal epithelial cells from apoptosis through activation of STAT3 ${ }^{62}$. We found that $\operatorname{Tg}-t R X R \alpha$ mice produced high level of serum IL-6 2 days after DSS treatment (Fig. 3e), in agreement with their rapid recovery from body weight loss induced by DSS treatment (Fig. 2d) and enhanced STAT3 activation (Fig. 2f). Together, these results revealed an important role of tRXRa in modulating inflammatory microenvironment and cytokine production, which likely accounts for its activation of STAT3 during colitis.

Myeloid tRXRa expression is necessary for STAT3 activation. Our observation that $T g$ - $t R X R \alpha$ mice could promote macrophage infiltration and cytokine production prompted us to determine the effect of tRXRa expression in myeloid cells. Thus we crossed $t R X R \alpha^{\text {flox }}$ mice with LysM-Cre mice that express Cre in myeloid cells ${ }^{63}$. For comparison, $R X R \alpha^{\text {flox }}$ mice were also crossed with LysM-Cre mice. These mice were initially analyzed for tumorigenesis using the AOM/DSS protocol. When $L y s M-t R X R \alpha$ mice were analyzed, we found that the average tumor size was much larger in this group of mice when compared to LysM-cre mice (Fig. 4a). Overexpression of RXRa in myeloid cells, however, did not apparently affect tumor load 
a

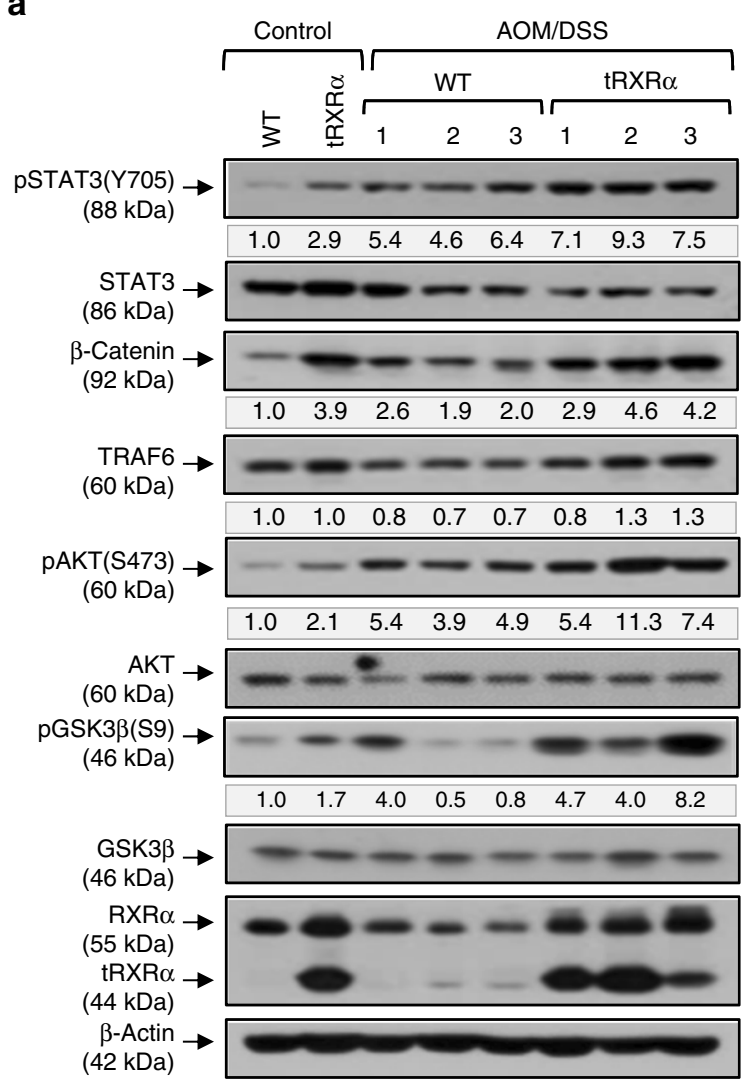

C
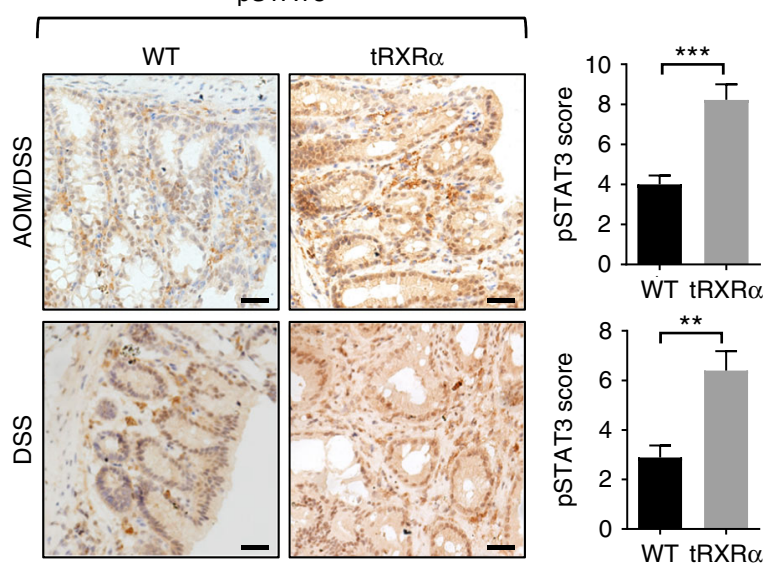

$\mathbf{f}$

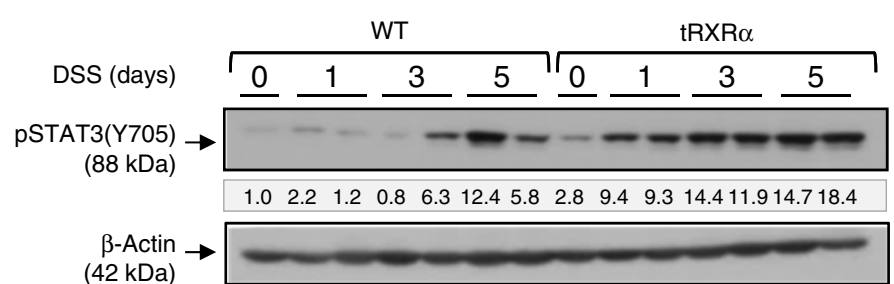

b

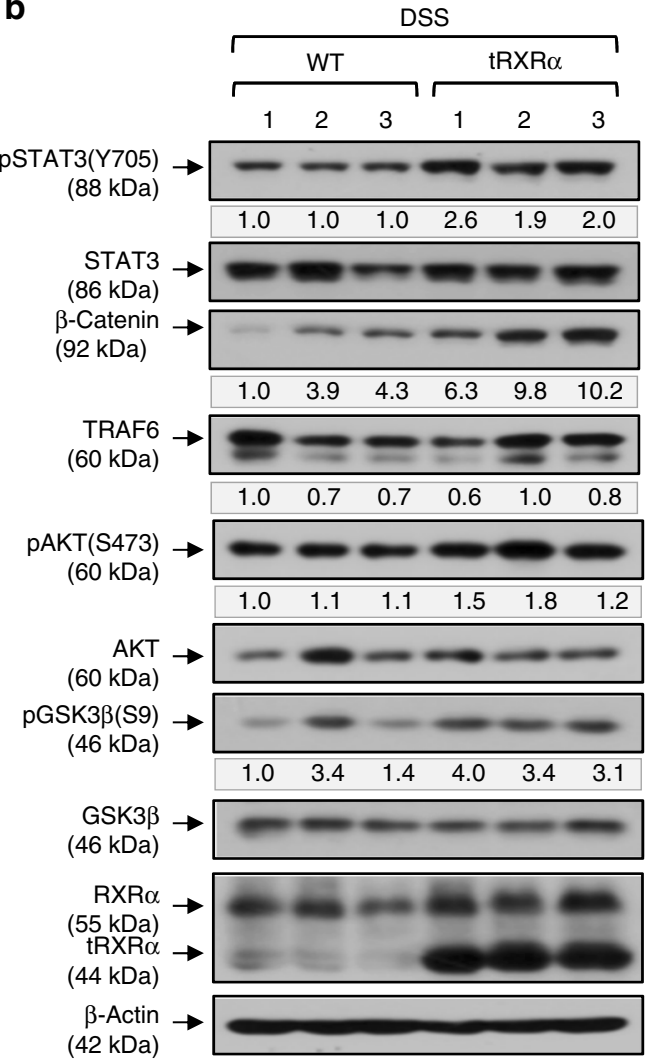

d

e

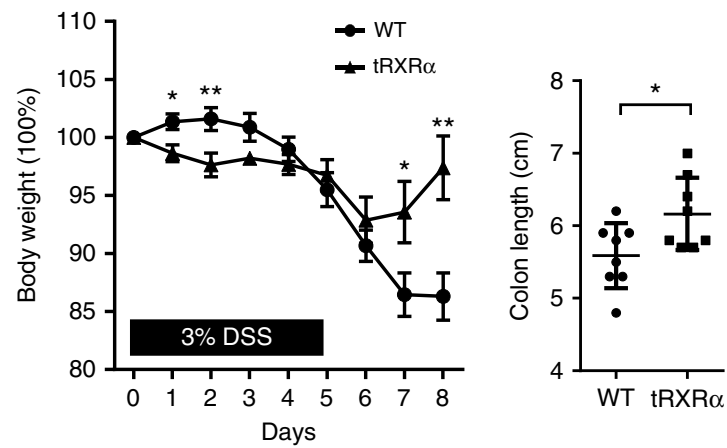

g

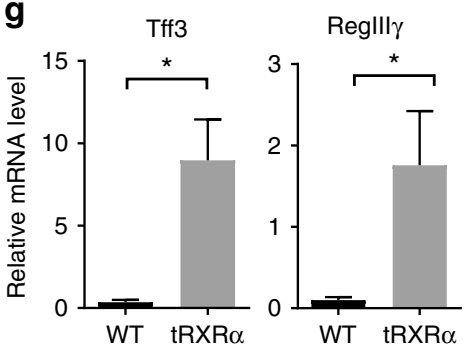

Fig. 2 Activation of signal transducer and activator of transcription factor 3 in colorectal tumors from Tg-tRXR $\alpha$ mice. a, b Colon lysates from mice treated with azoxymethane (AOM)/dextran sodium sulfate (DSS) for 120 days (a) or DSS for 6 months (b) were analyzed by immunoblotting. The bands are quantified with Image J and normalized to $\beta$-actin. c Colon sections from mice treated with AOM/DSS for 120 days or DSS for 6 months were stained with pSTAT3 antibody and analyzed the staining score. Scale bars, $25 \mu \mathrm{m}$. Data are mean \pm SEM $(n=9), t$ test. d Body weight curve of control and Tg-tRXR $\alpha$ mice during the course of acute DSS-induced colitis. Data are mean \pm SEM $(n=8)$, two-way analysis of variance. e Colon length from mice after the course of acute DSS-induced colitis. Data are mean \pm SD $(n=8), t$ test. $\mathbf{f}$ Colonic lysates prepared from mice treated with DSS for the indicated time were analyzed by immunoblotting. $\mathbf{g}$ Reglll $\gamma$ and Tff3 mRNA expression in mice treated with DSS for two days was analyzed by quantitative reverse transcriptase-PCR. Data are mean $\pm \operatorname{SEM}(n=3), t$ test. ${ }^{\star} P<0.05,{ }^{\star \star} P<0.01,{ }^{\star \star \star} P<0.001$. For immunoblotting, one of three or four similar experiments is shown 


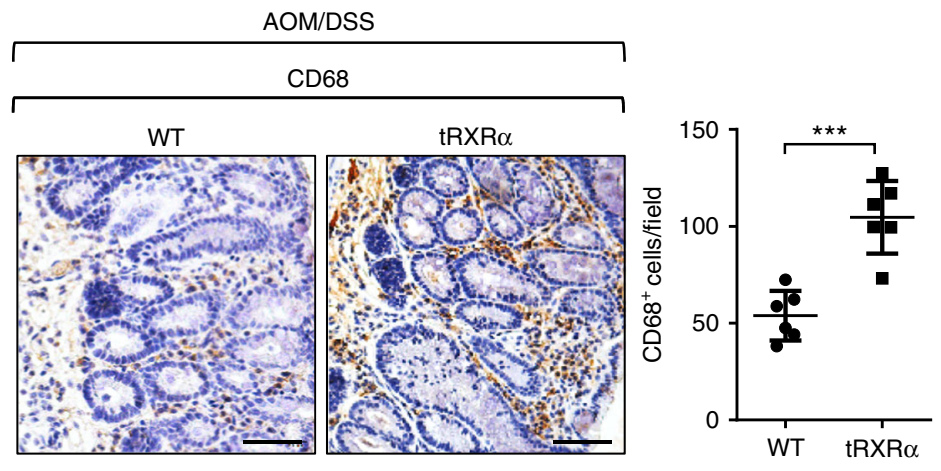

b
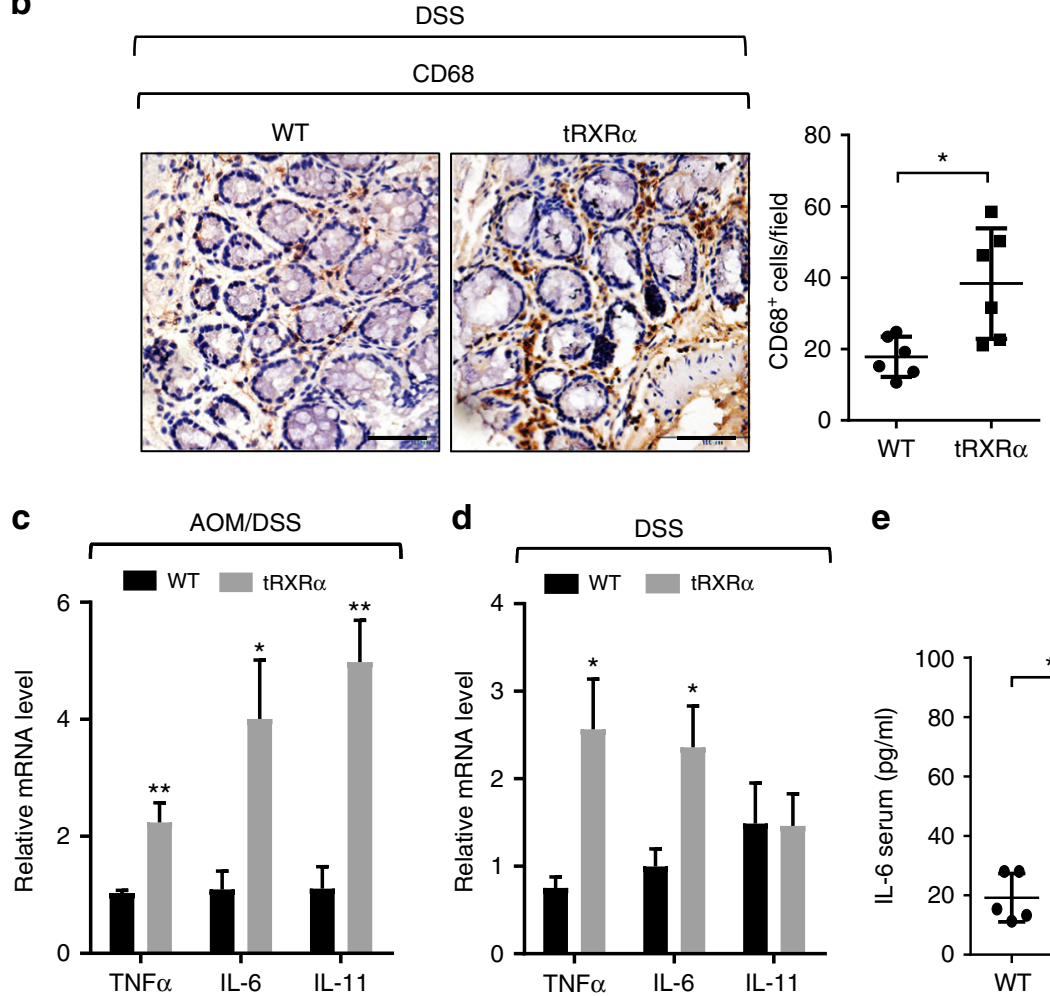

e

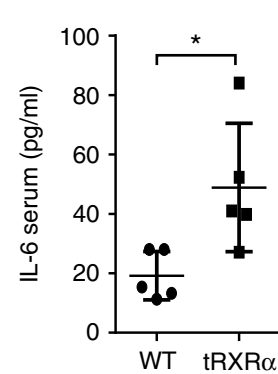

Fig. 3 Increased infiltration of inflammatory cells and cytokine production in $T g-t R X R \alpha$ tumor. $\mathbf{a}, \mathbf{b}$ Colonic sections from mice treated with azoxymethane $(A O M) /$ dextran sodium sulfate (DSS) (a) or DSS (b) were stained with CD68 antibody to detect macrophages and analyzed for the number of CD68positive cells per field (scale bars, $50 \mu \mathrm{m})$. Each dot represents a mouse, 10 fields were counted per mouse. Data are mean $\pm \operatorname{SD}(n=6), t$ test. $\mathbf{c}, \mathbf{d}$ Relative mRNA expression levels of the indicated genes in colon of mice treated with AOM/DSS (c) or DSS (d) were determined by quantitative reverse transcriptase-PCR. Data are mean \pm SEM $(n=4), t$ test. e Serum levels of interleukin- 6 from mice treated with $3 \%$ DSS for 2 days were measured by enzyme-linked immunosorbent assay. Each dot represents a mouse. Data are mean $\pm \mathrm{SD}(n=5), t$ test. ${ }^{\star} P<0.05,{ }^{\star \star} P<0.01,{ }^{\star \star \star} P<0.001$

as $L y s M-R X R \alpha$ mice showed similar tumor size and multiplicity with LysM-cre mice (Fig. 4a, b). Representative hematoxylin and eosin (H\&E) images revealed an increase in tumor size and high-grade dysplasia in $L y s M-t R X R \alpha$ mice (Fig. 4c). Thus tRXRa expression in myeloid cells could promote the growth of AOM/DSS-induced colorectal tumor. The role of tRXRa expression in myeloid cells was also illustrated by increased macrophage infiltration (Fig. 4d) and mRNA expression of IL-6, IL-11, and TNFa (Fig. 4e) in colon tumor tissues from $L y s M-t R X R \alpha$ but not in $L y s M-R X R \alpha$ mice. When the activation of STAT3 was examined, we found that it was strongly activated in $L y s M-t R X R \alpha$ but not in $L y s M-R X R \alpha$ mice (Fig. 4f), demonstrating that tRXRa expression in myeloid cells is responsible for STAT3 activation. Thus tRXRa expression in myeloid cells can induce IL-6 expression and STAT3 activation in colorectal tumor.
Activation of the IKK-NF-KB pathway by tRXRa in macrophages. To determine the molecular mechanism by which tRXR $\alpha$ expression in inflammatory cells promotes the growth of colorectal tumor through the IL-6-STAT3 pathway, we prepared bone marrow-derived macrophages (BMDMs) from $T g-t R X R \alpha$ mice (tRXRa-BMDMs) and the corresponding control wild-type mice (Supplementary Fig. 4a). tRXRa-BMDMs showed enhanced mRNA expression of IL- 6 and TNFa (Fig. 5a) and secretion of IL6 (Fig. 5b) in the absence or presence of lipopolysaccharide (LPS) when compared to control BMDMs, consistent with data obtained with $L y s M-t R X R \alpha$ mice (Fig. 4e). As IL-6 is a nuclear factor (NF)-kB-regulated cytokine in inflammatory cells ${ }^{61,62}$, we determined whether tRXRa could promote the activation of the IкB kinase (IKK)-NF- $\kappa B$ inflammatory pathway in macrophages. Treatment of control BMDMs with LPS resulted in IкBa degradation, an indicative of IKK activation, in a time- and dose- 
a

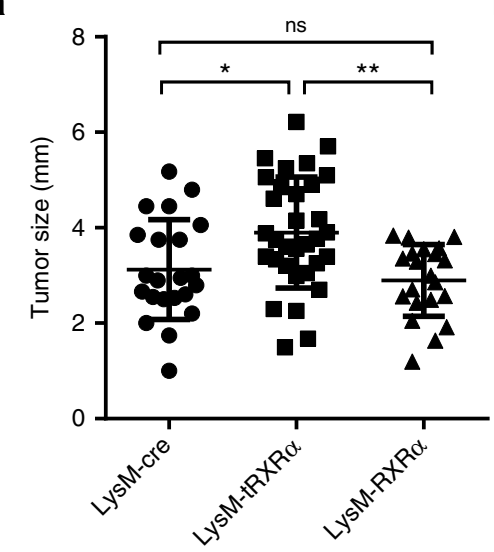

C

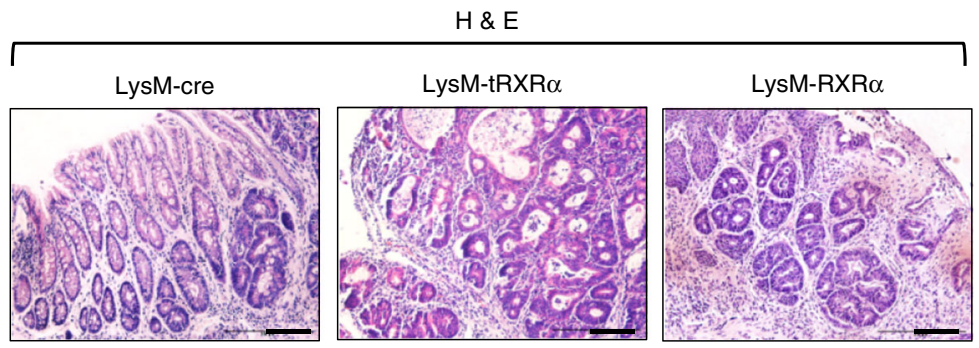

b Tumor size distribution

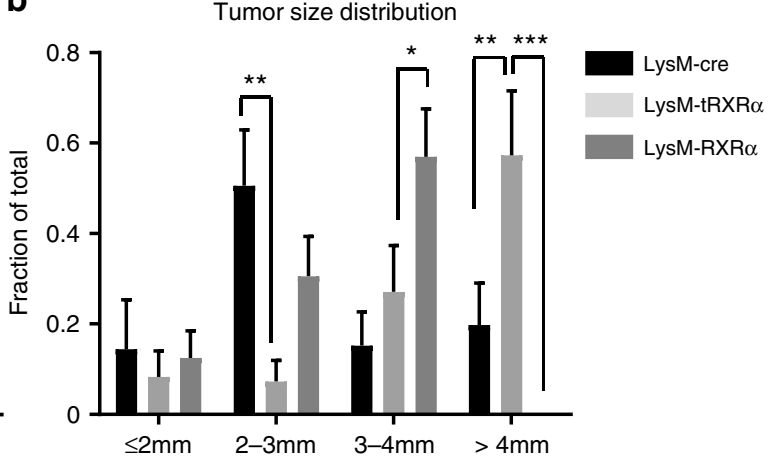

d

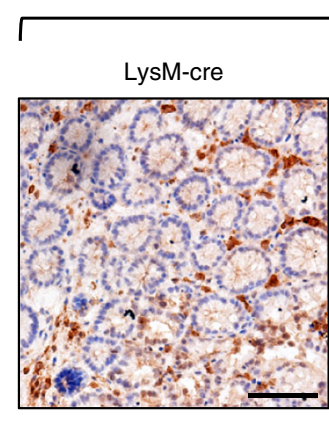

CD68
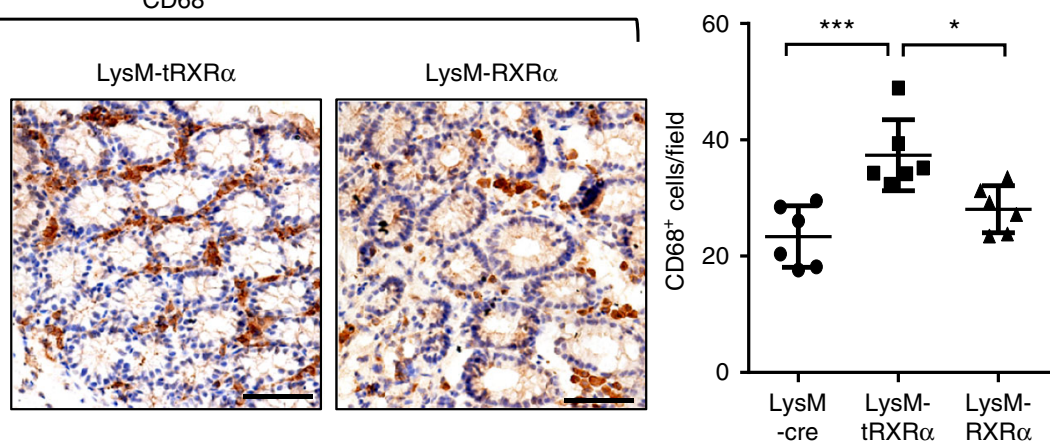

-cre $\quad \operatorname{tRXR} \alpha \quad \operatorname{RXR} \alpha$ e

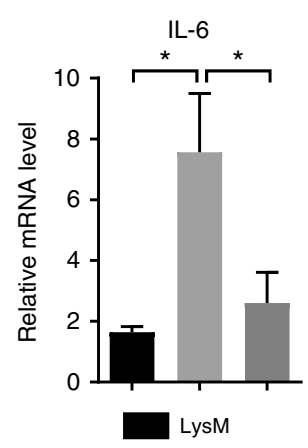

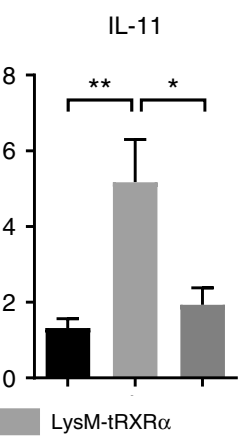

$\mathrm{TNF} \alpha$

f
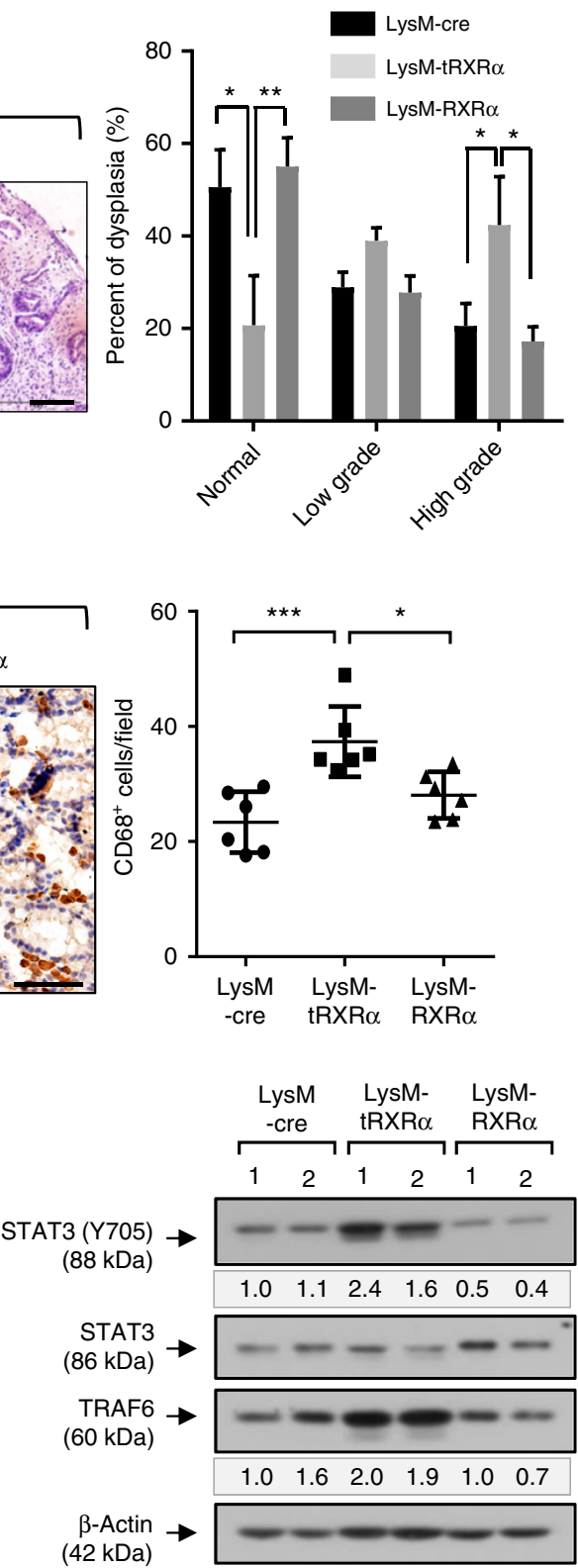

dependent manner (Fig. 5c). When tRXRa-BMDMs were analyzed, we found that the basal level of IкBa was much lower in tRXRa-BMDMs as compared to that in control BMDMs, suggesting a role of tRXRa in activating IKK in an LPS-independent manner. tRXRa could also potentiate the effect of LPS, as LPS- induced I $\mathrm{K} B \alpha$ degradation occurred much faster in tRXR $\alpha$ BMDMs than in control BMDMs. The role of tRXRa in activating the IKK-mediated inflammatory pathway was also supported by our data showing that stable expression of tRXRa in RAW264.7 cells (Supplementary Fig. 4b, c) enhanced the mRNA expression 
Fig. 4 Myeloid-specific truncated retinoid X receptor $\alpha$ expression is necessary for signal transducer and activator of transcription factor 3 activation and colorectal carcinogenesis. a Tumor size from mice treated with azoxymethane (AOM)/dextran sodium sulfate (DSS) for 4 months. Each dot represents a tumor. Data are mean \pm SD $(n=6)$, one-way analysis of variance (ANOVA). b Tumor size distribution. Data are mean \pm SEM ( $n=6)$, two-way ANOVA. c Colon tumor sections from mice treated with AOM/DSS for 4 months were stained with hematoxylin and eosin, Scale bars, $100 \mu \mathrm{m}$, and classified into normal, low-grade, and high-grade lesions based on histological analysis $(n=6)$. Data are mean \pm SEM $(n=6)$, two-way ANOVA. d Representative CD68 immunostaining image of colonic sections from mice treated with AOM/DSS (scale bars, $50 \mu \mathrm{m}$ ) and the score of the number of CD68-positve cells per field. Each dot represents a mouse, and 10 fields were counted per mouse. Data are mean $\pm \operatorname{SD}(n=6)$, one-way ANOVA. e Relative mRNA expression levels of the indicated genes in colon of mice treated with AOM/DSS were determined by quantitative reverse transcriptase-PCR. Data are mean \pm SEM $(n \geq 4)$. one-way ANOVA. $\mathbf{f}$ Colon lysates from mice treated with AOM/DSS were analyzed by immunoblotting. The bands are quantified with Image $\mathrm{J}$ and normalized to $\beta$-actin. One of three similar experiments is shown. ${ }^{\star} P<0.05,{ }^{\star \star} P<0.01,{ }^{\star \star \star} P<0.001$
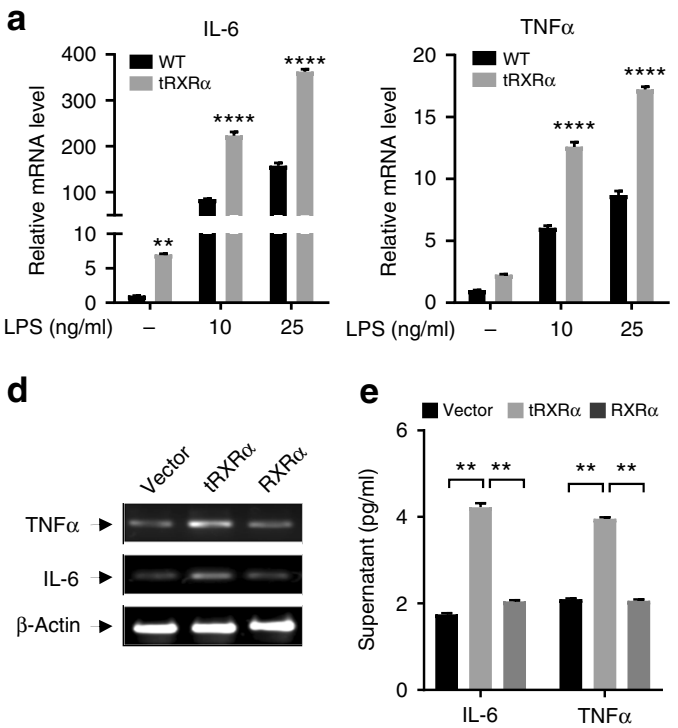
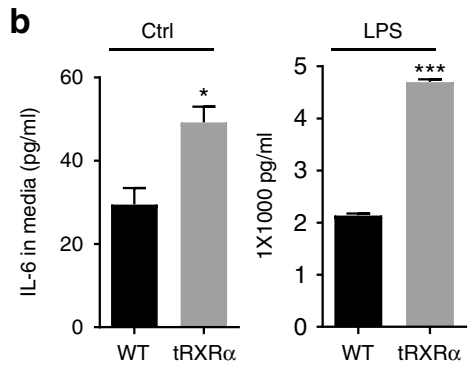

f

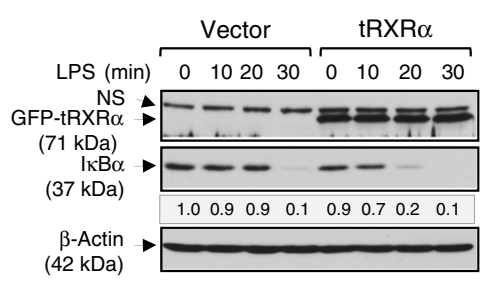

C

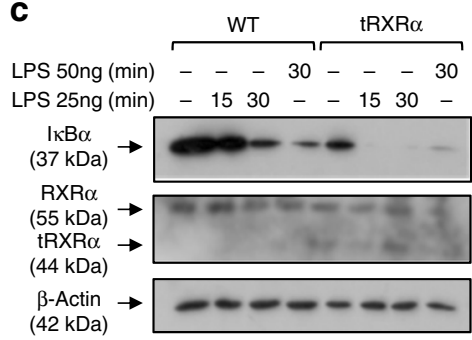

g

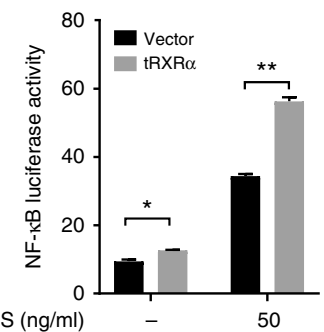

h

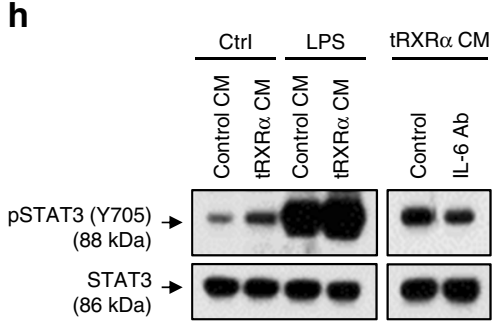

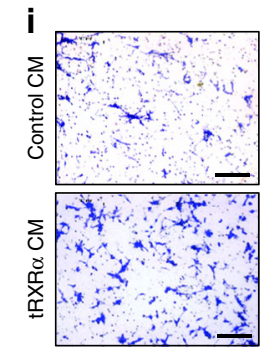
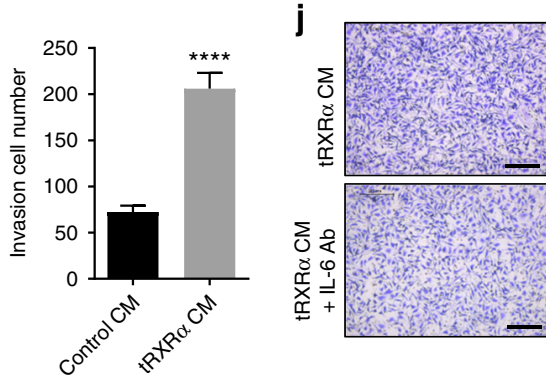

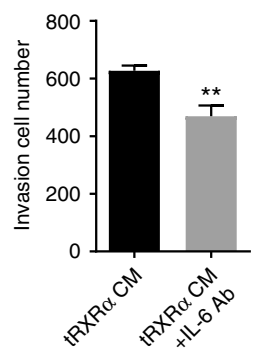

Fig. 5 Truncated retinoid X receptor $\alpha(\operatorname{tRXR} \alpha)$ activation of the IKK-NF- $\mathrm{kB}$ pathway in macrophages. a Relative mRNA expression of interleukin (IL)-6 and tumor necrosis factor- $\alpha$ (TNF $\alpha$ ) in wild-type (WT) and tRXR $\alpha$-BMDMs treated with or without lipopolysaccharide (LPS) for $3 \mathrm{~h}$ was determined by quantitative reverse transcriptase-PCR (qRT-PCR), two-way analysis of variance (ANOVA). b WT and tRXR $\alpha$-BMDMs cultured with Dulbecco's modified Eagle's medium containing $10 \%$ fetal bovine serum for 3 days or treated with LPS $(25 \mathrm{ng} / \mathrm{ml})$ for $12 \mathrm{~h}$ were analyzed by enzyme-linked immunosorbent assay (ELISA), $t$ test. c Immunoblotting of lysates from WT and tRXR $\alpha$-BMDMs treated with or without LPS for the indicated time. $\mathbf{d}$ Expression of IL-6 and TNF $\alpha$ mRNAs in RAW264.7 cells stably expressing green fluorescent protein (GFP), GFP-tRXR $\alpha$, and GFP-RXR $\alpha$ was analyzed by RT-PCR. e Production of IL-6 and TNF $\alpha$ in RAW264.7 cells stably expressing GFP, GFP-tRXR $\alpha$, and GFP-RXR $\alpha$ was analyzed ELISA, one-way ANOVA. f Stable expression of tRXR $\alpha$ in RAW264.7 cells potentiate LPS-induced I $\mathrm{kB} \alpha$ degradation determined by immunoblotting. NS nonspecific. $\mathbf{g}$ Stable expression of tRXR $\alpha$ in RAW264.7 cells promotes LPS-induced NF-KB transactivation determined by reporter assay, two-way ANOVA. $\mathbf{h}$ Condition medium (CM) collected from WT- and tRXR $\alpha$-BMDMs cultured for 3 days and treated with or without LPS $(25 \mathrm{ng} / \mathrm{ml})$ for $12 \mathrm{~h}$ was used to culture mouse CT26 colon cancer cells for $1 \mathrm{~h}$ and analyzed for signal transducer and activator of transcription factor 3 activation. Anti-IL- 6 antibody (Ab) was incubated with CM of tRXR $\alpha$-BMDMs for $1 \mathrm{~h}$ before being used to culture CT26 cells. i CT26 cells were cultured with CM collected from WT- or tRXR $\alpha$-BMDMs for $24 \mathrm{~h}$ in transwell and analyzed by invasion assay. Representative images were photographed and migrated cells per field were counted. Scale bar, $200 \mu \mathrm{m}, t$ test. $\mathbf{j}$ CM from tRXR $\alpha$-BMDMs was incubated with or without IL- 6 antibody and used to culture CT26 cells for $36 \mathrm{~h}$. Representative images were photographed and migrated cells per field were counted. Scale bar, $200 \mu \mathrm{m},{ }^{\star \star} P<0.01$ by $t$ test. Data are mean $\pm \mathrm{SEM},{ }^{\star} P<0.05,{ }^{\star \star} P<0.01,{ }^{\star \star \star} P<0.001,{ }^{\star \star \star} P<0.0001$. For immunoblotting, one of three or four similar experiments is shown 
a

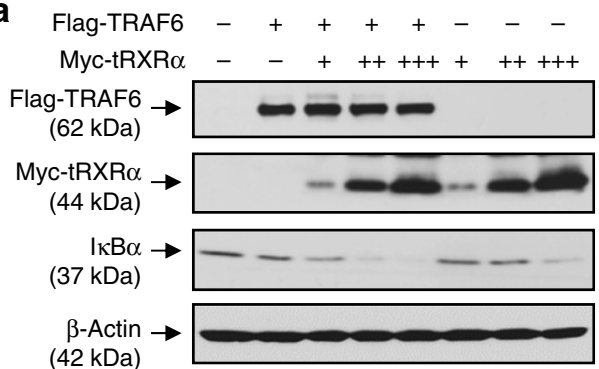

b

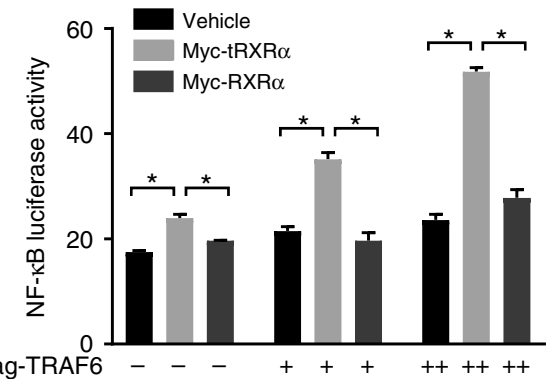

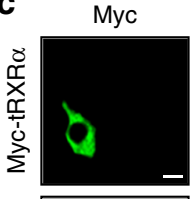

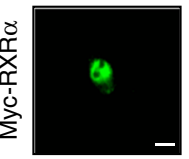

d

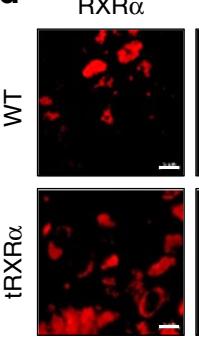

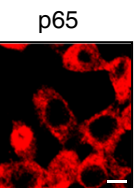
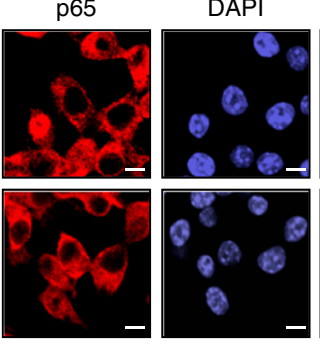

Macrophage

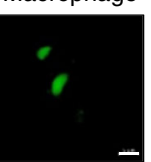

DAPI

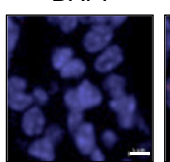

Merge
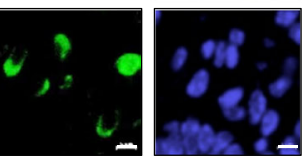

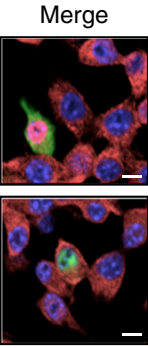

e
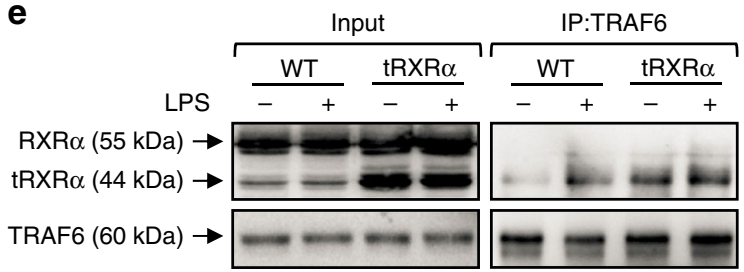

f

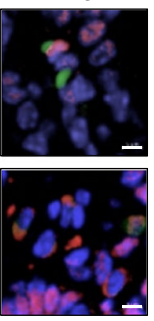

g

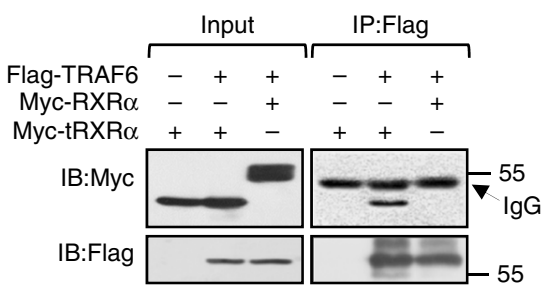

h
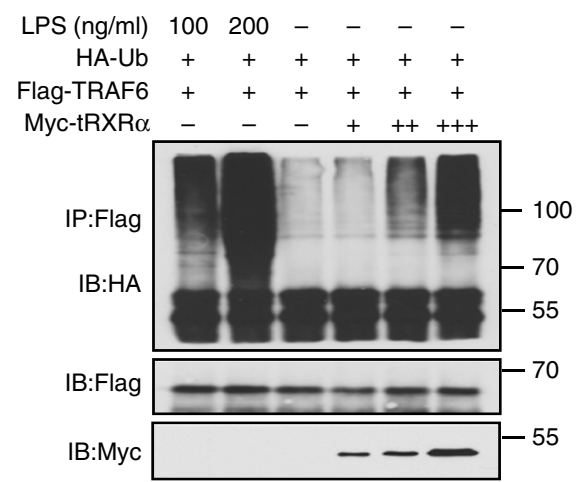

i

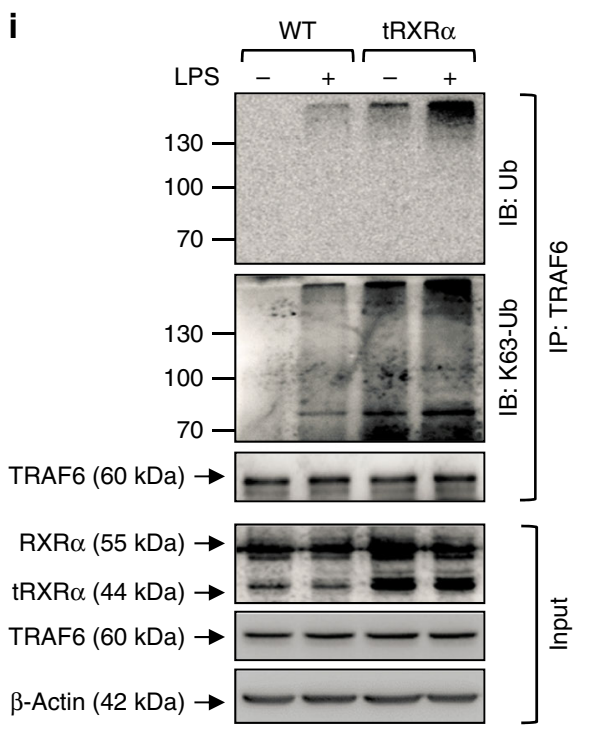

of IL-6 and TNFa (Fig. 5d) and their secretion in medium (Fig. 5e), and the effect of LPS on inducing IKB $\alpha$ degradation (Fig. 5f) and NF-KB transactivation (Fig. 5g). Conversely, transfection of RXRa small interfering RNA (siRNA) in THP-1 cells, which inhibited the expression of both RXR $\alpha$ and tRXRa, attenuated the effect of LPS on inducing I $\kappa$ Ba degradation (Supplementary Fig. 4d) and activating the NF- $\mathrm{KB}$ reporter gene transcription (Supplementary Fig. 4e). LPS-induced mRNA expression of IL- 6 and TNFa was also inhibited by RXRa siRNA transfection (Supplementary Fig. 4f). Collectively, these results demonstrated that tRXRa is involved in regulating the IKK-NF$\kappa B$ pathway in macrophages.

We next studied whether tRXR $\alpha$ activation of the IKK-NF- $\mathrm{kB}$ inflammatory pathway in macrophages could serve to activate STAT3 in colon cancer cells. Thus condition medium (CM) from tRXRa-BMDMs were prepared and used to culture mouse CT26 colon cancer cells. Analysis of STAT3 activation in CT26 cells revealed that CM from tRXRa-BMDMs was much more effective than control BMDMs in activating STAT3 (Fig. 5h). Cell invasion assays showed that the invasion of CT26 cells was significantly 
Fig. 6 Truncated retinoid X receptor $\alpha(t R X R \alpha)$ interacts with TRAF6 and activates the IKK-NF- $\mathrm{kB}$ pathway. a Lysates from HEK293T cells transfected with Flag-TRAF6 and/or increasing amounts of Myc-tRXR $\alpha$ were analyzed by immunoblotting. $\mathbf{b}$ CV-1 cells transfected with pNF-kB reporter alone or in combination with RXR $\alpha$ or TRAF6 plasmids were analyzed for nuclear factor (NF)- $\kappa B$ transactivation by reporter assay. Data are mean \pm SEM, ${ }^{\star} P<0.05$, by two-way analysis of variance. c Subcellular localization of transfected Myc-tRXR $\alpha$ or Myc-RXR $\alpha$ in RAW264.7 cell was revealed by immunostaining using anti-Myc antibody. The localization of endogenous p65 was stained by anti-p65 antibody. Green, Myc; red, p65; blue, DAPI. Scale bar, $10 \mu \mathrm{m}$. d Subcellular localization of RXR $\alpha$ in macrophages of colon tumor tissues from mice treated with azoxymethane/dextran sodium sulfate for 120 days was determined by immunostaining using $\Delta$ N197 anti-RXR $\alpha$ antibody. Green, CD68; Red, $\Delta$ N197; Blue, DAPI. Scale bar, $5 \mu$ m. e Wild-type (WT)- and tRXR $\alpha$-BMDMs treated with lipopolysaccharide (LPS; $50 \mathrm{ng} / \mathrm{ml}$ ) for $15 \mathrm{~min}$ were analyzed for tRXR $\alpha$ interaction with TRAF6 by immunoprecipitation assays using anti-TRAF6 antibody. Immunoprecipitates were analyzed by immunoblotting. f, g HEK293T cells transfected with the indicated plasmids were analyzed by coimmunoprecipitation assays using anti-Myc or anti-Flag antibody. h RAW264.7 transfected with HA-Ub, Flag-TRAF6, and Myc-tRXR $\alpha$ plasmids were immunoprecipitated with anti-Flag antibody and analyzed for TRAF6 ubiquitination by immunoblotting. i WT- and tRXR $\alpha$-BMDMs treated with or without LPS $(50 \mathrm{ng} / \mathrm{ml})$ for $15 \mathrm{~min}$ were immunoprecipitated with anti-TRAF6 antibody and analyzed for TRAF6 ubiquitination by immunoblotting. For immunoblotting, one of three or four similar experiments is shown
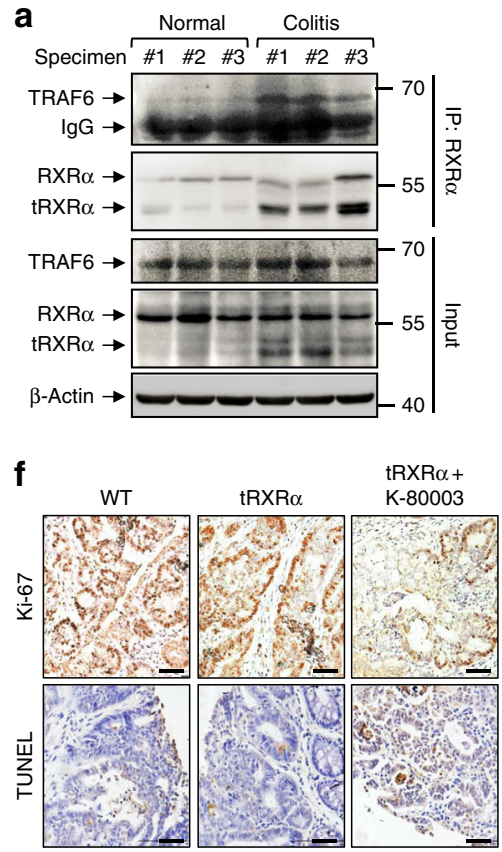

b
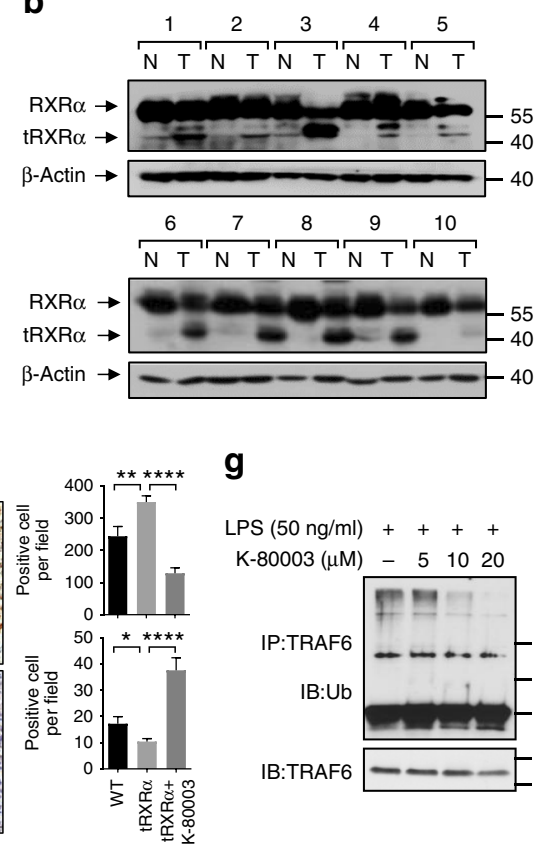

g

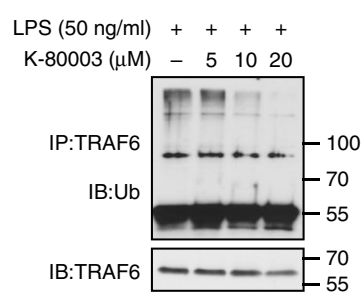

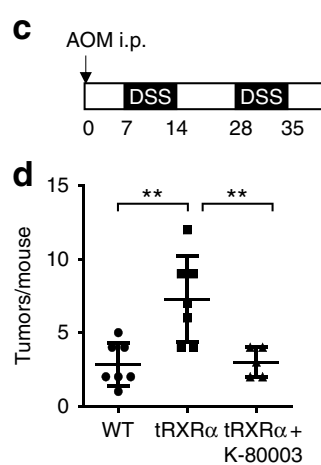

$\mathbf{h}$

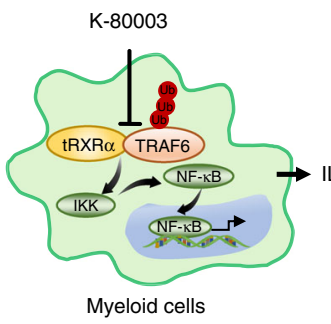

K-80003

nunuming
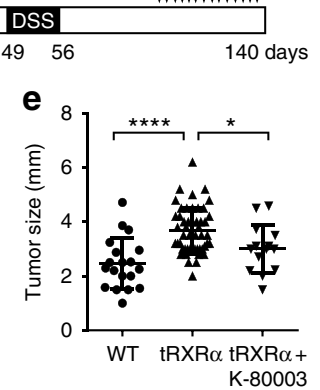

Fig. 7 Expression of truncated retinoid $X$ receptor $\alpha(\operatorname{tRXR} \alpha)$ in human specimens and the inhibitory effect of $\mathrm{K}-80003$ on tRXR $\alpha$-mediated colorectal carcinogenesis. a tRXR $\alpha$ expression and interaction with TRAF6 in ulcerative colitis patients. Lysates of colorectal tissues from healthy and ulcerative colitis patients were analyzed by immunoblotting and co-immunoprecipitation assay using $\Delta N 197$ anti-RXR $\alpha$ antibody. $\mathbf{b}$ The expression of RXR $\alpha / t R X R \alpha$ were immunoblotting in tumor tissue $(T)$ and corresponding adjacent normal tissue $(N)$ from 10 colorectal tumor patients. c Schematic representation of the azoxymethane (AOM)/dextran sodium sulfate (DSS) protocol and K-80003 treatment. Mice were treated with AOM/DSS for 125 days and then administered with vehicle control or $\mathrm{K}-80003(20 \mathrm{mg} / \mathrm{kg})$ via oral gavage for 2 weeks daily. d Average tumor number. Data points represent the tumor number of one individual mouse. The horizontal bar represents the mean \pm SD $(n=7 / 5)$, one-way analysis of variance (ANOVA). e Average tumor size. Each dot represents a tumor. The horizontal bar represents the mean \pm SD, one-way ANOVA. $\mathbf{f}$ Representative images of colon cancer stained with Ki-67 antibody or with the terminal deoxinucleotidyl transferase-mediated dUTP-fluorescein nick end labeling (TUNEL) Kit. Ki67-positive or TUNEL-positive cells per field were counted. Data are mean \pm SEM, one-way ANOVA. Scale bar, $100 \mu \mathrm{m}$. $\mathbf{g}$ THP-1 cells pretreated with the indicated concentrations of K-80003 before exposed to lipopolysaccharide $(50 \mathrm{ng} / \mathrm{ml})$ were subjected to immunoprecipitation assay using anti-TRAF6 antibody and analyzed for TRAF6 ubiquitination by immunoblotting. $\mathbf{h}$ Schematic of tRXR $\alpha$ activation of the nuclear factor- $\mathrm{kB}$ pathway in macrophages and its role in promoting colon cancer cell survival and proliferation through induction of signal transducer and activator of transcription factor 3 activation. ${ }^{\star} P<0.05,{ }^{\star \star} P<0.01$, ${ }^{\star \star \star} P<0.001$. For immunoblotting, one of three or four similar experiments is shown

increased when they were cultured with CM from tRXRaBMDMs but not from control BMDMs (Fig. 5i). CM from tRXRa-BMDMs could also potentiate the effect of LPS on activating STAT3. Similar results were obtained when THP-1 cells were analyzed. In this case, transfection of tRXR $\alpha$ but not of RXRa strongly induced the expression of IL-6 and TNFa (Supplementary Fig. 5a). CM from tRXRa-transfected but not from RXR $\alpha$-transfected THP-1 cells enhanced STAT3 activation in human HCT-116 colon cancer cells (Supplementary Fig. 5b). In agreement with enhanced STAT3 activation, cell invasion assays showed that the invasion of colon cancer cells was significantly increased when they were cultured with CM from tRXRa-BMDMs (Fig. 5i) or co-cultured with THP-1 cells expressing tRXRa but not RXRa (Supplementary Fig. 5c). Interestingly, tRXR $\alpha$-expressing THP-1 cells could also promote the expression of c-Myc and cyclin D1 and growth and invasion of breast cancer cells (Supplementary Fig. 5d-f), indicating that the effect of tRXRa in macrophages is not limited to colon cancer. To determine whether IL- 6 secreted from tRXR $\alpha$-BMDMs played a role in STAT3 activation, CM from tRXRa-BMDMs was 
incubated with anti-IL-6 antibody. Neutralization of IL-6 activity by anti-IL-6 antibody diminished its effect on inducing STAT3 activation in CT26 cells (Fig. 5h) and their invasion activity (Fig. 5j), revealing a role of IL-6 in STAT3 activation and colon cancer cell growth.

tRXRa interacts with TRAF6 in macrophages. TRAF6, an essential upstream regulator of the IKK complex, is one of the key signaling proteins in the IKK/NF- $\mathrm{BB}$ pathway in macrophages ${ }^{64,65}$. TRAF6 is highly expressed in many different types of cancer including colorectal cancer ${ }^{66,67}$. Interestingly, the levels of TRAF6 were significantly elevated in $T g-t R X R \alpha$ mice challenged with either AOM/DSS (Fig. 2a) or DSS (Fig. 2b). Colon tumor from $L y s M-t R X R \alpha$ mice also showed a significant elevated level of TRAF6 expression, which positively correlated with STAT3 activation (Fig. 4f). These observations promoted us to study whether and how TRAF6 mediated the effect of tRXR $\alpha$ on activating the NF-kB-IL-6-STAT3 cascade. Indeed, cotransfection of TRAF6 augmented the effect of tRXR $\alpha$ on inducing I $\mathrm{B} \alpha$ degradation (Fig. 6a) and NF- $\kappa B$ transactivation (Fig. 6b), suggesting a role of TRAF6 in mediating tRXR $\alpha$ activity.

TRAF6 activation of the IKK complex occurs in the cytoplasm ${ }^{64,65}$. As the first step to study whether tRXRa interacted with TRAF6, we examined the subcellular localization of tRXRa in RAW246.7 cells and found that transfected MyctRXR $a$ resided in the cytoplasm while transfected Myc-RXRa was in the nucleus (Fig. 6c). Immunostaining also revealed predominant cytoplasmic RXRa staining in colon tumor from $\mathrm{Tg}$ $t R X R \alpha$ mice (Fig. 6d). Thus tRXRa is a cytoplasmic protein in macrophage. We then tested whether tRXR $a$ could interact with TRAF6 in the cytoplasm to modulate IKK activation. Immunoprecipitation of TRAF6 from tRXRa-BMDMs resulted in coimmunoprecipitation of $\operatorname{tRXR} \alpha$, which was slightly enhanced when cells were treated with LPS (Fig. 6e). The interaction appears to be very stable as trace amount of tRXRa expressed in control BMDMs also interacted with TRAF6 in an LPSdependent manner. The LPS-dependent interaction between endogenous TRAF6 and tRXR $\alpha$ was also observed in THP-1 and RAW264.7 cells (Supplementary Fig. 6a). The interaction between TRAF6 and tRXRa was confirmed by data showing that transfected Myc-tRXRa but not Myc-RXRa interacted with transfected Flag-TRAF6 (Fig. 6f, g). Our mutagenesis analysis revealed that the interaction involved the TRAF domain of TRAF6 and the LBD of tRXRa (Supplementary Fig. 6b-d).

TRAF6 has E3 ligase activity, which can induce autoubiquitination necessary for its activation of $\mathrm{IKK}^{64}$. We thus determined whether tRXRa interaction with TRAF6 could result in TRAF6 autoubiquitination. Consistent with previous results ${ }^{68}$, transfected Flag-TRAF6 was strongly ubiquitinated by LPS. Similar to the effect of LPS, transfection of tRXRa promoted TRAF6 ubiquitination in a dose-dependent manner (Fig. 6h). In contrast, transfection of RXRa had no such an effect (Supplementary Fig. 6e). Stable expression of tRXRa in RAW264.7 cells (Supplementary Fig. 6f) or tRXRa expression in BMDMs (Fig. 6i) could potentiate the effect of LPS on inducing TRAF6 ubiquitination. Taken together, these results demonstrated that tRXRa can activate the IKK-NF- $\mathrm{KB}$ pathway in macrophages by inducing TRAF6 autoubiquitination through their direct protein-protein interaction.

To study whether tRXR $\alpha$ production and interaction with TRAF6 is clinic significant, we examined the expression of tRXR $\alpha$ in tissue specimens from patients with ulcerative colitis and colorectal cancer. Our results demonstrated that tRXR $\alpha$ was expressed in colorectal tissues from three ulcerative colitis patients while tRXR $\alpha$ was barely detected in colorectal tissues from three healthy individuals (Fig. 7a). In addition, various degrees of tRXRa expression were found in tumor tissues but not the corresponding adjacent normal tissues from ten colon cancer patients examined (Fig. 7b). Moreover, tRXRa expressed in ulcerative colitis patients could interact with TRAF6. Thus tRXR $a$ production and interaction with TRAF6 appears to have role in the pathogenesis of CAC.

Inhibition of the tumorigenic effect of tRXRa by K-80003. K80003 is a potent inhibitor of tRXR $\alpha$ through its unique tRXR $\alpha$ binding mechanism ${ }^{52}$. Administration of $T g-t R X R \alpha$ mice with $\mathrm{K}$ 80003 during the late stage of AOM/DSS-induced colorectal carcinogenesis (Fig. 7c) significantly reduced colon tumor load, reflected by both smaller tumor sizes and less tumor numbers as compared to vehicle-treated mice (Fig. 7d, e; Supplementary Fig. 7a, b). The potent anti-CAC effect of K-80003 was associated with its inhibition of cell proliferation and induction of apoptosis (Fig. 7f), suppression of the mRNA expression of IL-6, TNFa, and IL-11 and the serum production of IL-6, and inhibition of STAT3 activation (Supplementary Fig. 7c-e). These data demonstrated that $\mathrm{K}-80003$ is a potent inhibitor of tRXRa-mediated IL-6STAT3 signaling during colorectal tumorigenesis. In vitro, K80003 inhibited LPS induction of mRNA expression of IL-6 (Supplementary Fig. 7f) in a dose-dependent manner in macrophages. It also suppressed the effect of LPS on inducing I $\kappa B \alpha$ degradation, NF- $\mathrm{KB}$ transactivation, and nuclear translocation (Supplementary Fig. 7g-i) in RAW264.7 cells. Mechanistically, K80003 inhibited LPS-induced interaction of tRXRa with TRAF6 (Supplementary Fig. 7j) and TRAF6 ubiquitination (Fig. 7g). Together, these results demonstrated that K-80003 is a potent inhibitor of tRXR $\alpha$-mediated CAC development by preventing tRXR $\alpha$ interaction with TRAF6.

\section{Discussion}

Our characterization of the $t R X R \alpha$ transgenic mice revealed a critical role of tRXR $\alpha$ in the development of CAC. Tg-tRXR $\alpha$ mice displayed increased tumor frequency and enlarged tumor size when applied to the AOM/DSS protocol (Fig. 1). Colorectal tumors developed from $T g$ - $t R X R \alpha$ mice showed much more profound macrophage infiltration and expressed high levels of inflammatory cytokines (Fig. 3), demonstrating that the tumorigenic effect of tRXR $\alpha$ is due to its modulation of the tumor inflammatory microenvironment. This was supported by our discovery that $\mathrm{tRXR} \alpha$ expression in myeloid cells could promote colorectal carcinogenesis (Fig. 4) and is in agreement with previous reports that RXR $\alpha$ plays an important role in regulating macrophage functions and inflammatory disorders ${ }^{33,35}$. The role of tRXRa in myeloid cells was further illustrated by our in vitro experiments showing that ectopic expression of tRXRa but not of RXR $\alpha$ in macrophages potentiated LPS induction of I $\mathrm{KB} \alpha$ degradation, NF- $\mathrm{KB}$ nuclear translocation, and expression of several cytokines (Fig. 5). Thus the tumorigenic role of tRXRa during CAC development is largely attributed to its activation of the IKK-NF- $\kappa B$ pathway in macrophages. Our observations that tRXRa is produced in colorectal tissues of ulcerative colitis patients (Fig. 7a) and a majority of tumor tissues but not the corresponding adjacent normal tissues from patients with colorectal tumor (Fig. 7b), suggest the clinical relevance of tRXRa expression in the development of CAC.

Recent studies have provided important insight into the molecular mechanisms linking chronic inflammation to the development of cancers. Of particular important mechanism is the activation of STAT3 by IL- $6^{61,62}$. However, the regulation of the IL-6-STAT3 pathway during the development of CAC is still obscure, which has hindered the development of effective 
strategies targeting the pathway. Our present data demonstrated that tRXRa is an important modulator of the IL-6-STAT3 cascade during colorectal tumorigenesis. Enhanced STAT3 phosphorylation was observed in Tg-tRXR $\alpha$ mice before (Fig. 2a) or after treatment with AOM/DSS (Fig. 2a) or DSS alone (Fig. 2b). Induction of STAT3 phosphorylation by tRXRa was fast, occurring in 1 day after DSS treatment (Fig. 2f), suggesting a pivotal role of tRXRa in the activation of STAT3. TNFa, another cytokine known to activate the NF- $\kappa \mathrm{B}$ pathway, whose activation contributes to tumor development, was also upregulated in $t R X R \alpha$ mice (Fig. 3). TNFa activation of the NF- $\kappa B$ signaling induces the expression of inflammatory mediators and growth factors. We previously reported that tRXRa could promote TNFa activation of the PI3K/AKT signaling pathway, another important pathway critical for cancer cell proliferation and survival ${ }^{25}$. AKT activation was indeed enhanced in tRXRa transgenic mice challenged with AOM/DSS (Fig. 2a) or DSS (Fig. 2b). Taken together, these results identified tRXR $\alpha$ as an important factor that mediates the causal link between chronic inflammation and the development of CAC.

While how RXRa binds to DNA and regulates target gene transcription is well understood, the non-transcriptional actions regulated by RXRa and the underlying mechanisms remain poorly understood. Given the fact that transcription-independent action of RXRa and other nuclear receptors often involves their interaction with important signal transduction proteins critical for the growth and death of cancer cells, understanding the molecular mechanism by which nuclear receptors exert their nontranscriptional action may offer new strategies to explore their therapeutic potential. One of the unique properties of tRXR $\alpha$ is its cytoplasmic localization under inflammatory conditions ${ }^{9}$. Our investigation of the molecular mechanism by which tRXRa activates the inflammatory pathway in macrophages revealed a previously unrecognized non-transcriptional role of tRXRa through its interaction with TRAF6. TRAF6 is overexpressed in a variety of cancers including colon cancer ${ }^{66,67}$. TRAF6 overexpression in NIH3T3 cells resulted in NF- $\kappa B$ activation, anchorageindependent growth, and tumor formation, whereas TRAF6 depletion inhibited cancer cell proliferation and tumorigenesis ${ }^{69}$, demonstrating its oncogenic activity. As TRAF6 plays a key role in the assembly of protein complexes necessary for the activation of the IKK-NF- $\kappa \mathrm{B}$ pathway, TRAF6 overexpression would lead to constitutive NF- $\kappa \mathrm{B}$ activation. Indeed, we found that TRAF6 expression was enhanced in $T g$ - $t R X R \alpha$ mice in the absence or presence of AOM/DSS treatment (Fig. 2a). Moreover, TRAF6 expression was increased when tRXRa was expressed in myeloid cells (Fig. 4f). While how tRXRa induces TRAF6 expression in myeloid cells is currently unknown, we showed that coexpression of TRAF6 and tRXRa synergistically induced I $\mathrm{B} a$ degradation (Fig. 6a) and NF- $\kappa B$ activation (Fig. 6b). Such an effect was likely due to their physical interaction (Fig. 6), which led to autoubiquitination of TRAF6, an event known to activate the IKK/ NF- $\kappa B$ pathway ${ }^{64}$. Interestingly, TRAF6 interacted with tRXR $\alpha$ but not with $\mathrm{RXR} \alpha$, demonstrating that $\mathrm{tRXRa}$ activation of the IKK-NF- $\kappa B$ pathway is a tumor-specific event. Thus tRXR $\alpha$ activation of the NF- $\mathrm{B}$ pathway in macrophages through its interaction with cytoplasmic TRAF6 represents an important non-transcriptional mechanism by which $\mathrm{tRXR} \alpha$ promotes tumor growth.

Our discovery that tRXR $\alpha$ plays a pivotal role in modulating the IL-6-STAT3 pathway offers an effective and selective approach to treat inflammation-associated cancers. NSAIDs have demonstrated promising preventive and therapeutic effects against colorectal cancer. Sulindac, one of the most studied NSAIDs, is effective against colorectal cancer in various clinical studies ${ }^{53,54}$. Unfortunately, long-term sulindac treatment has several drawbacks, such as gastrointestinal bleeding associated with COX1 inhibition and increased cardiovascular risk resulting from COX2 inhibition ${ }^{70}$. Our previous discovery that tRXRa serves as an intracellular target of sulindac led to our identification of K-80003, a sulindac analog with enhanced tRXRa binding and diminished COX inhibition activities ${ }^{25}$. We showed here that $\mathrm{K}-80003$ is a potent inhibitor of tRXRa-mediated colorectal carcinogenesis by inhibiting tRXR $\alpha$-mediated NF- $\mathrm{BB}-\mathrm{IL}-6-\mathrm{STAT3}$ pathway (Fig. 7). The effect of K-80003 is likely due to its ability to induce tRXRa tetramerization ${ }^{52}$ that may serve to inhibit tRXR $\alpha$ interaction with TRAF6. It is noteworthy that K-80003 exhibits very desirable pharmacological profiles including much reduced side effects in animals ${ }^{25}$, which could be attributed to its effective and selective induction of tetramerization of tRXRa but not of RXRa ${ }^{52}$. The selective effect of $\mathrm{K}-80003$ is expected to lead to specific blockade of tRXRa in tumor cells without compromising the normal function of RXRa. The ability of K-80003 to bind to distinct and alternate hydrophobic grooves on the surface of tRXRa ${ }^{52}$ may also contribute to its efficacy and selectivity, as tRXRa may employ its surface grooves to interact with nontranscriptional signaling proteins such as TRAF6.

Together, our results revealed an oncogenic effect of tRXRa in the development and growth of CAC through its activation of the IL-6-STAT3 signaling pathway and uncovered a new tRXRa nontranscriptional mechanism responsible for the activation. Our data also identify K-80003 as a promising anti-CAC agent that targets the tRXR $\alpha$ pathway.

\section{Methods}

Mice. $\operatorname{Lox} P-t R X R \alpha$ and $\operatorname{lox} P-R X R \alpha$ mice were constructed by using pronuclear microinjection. The detail sequences are presented in Supplementary Fig. 1a and the sequence of $\operatorname{loxp}-t R X R \alpha$ are the same with $\operatorname{loxp}-R X R \alpha$ except that $85 \mathrm{~N}$ terminal amino acids in RXR $\alpha$ were deleted in loxp-tRXR $\alpha$. CMV-Cre mice (B6.C$\operatorname{Tg}(C M V$-cre $) 1 C g n / J)$ (Cat.J006054) and LysM-cre mice (B6.129P2-Lyz2tm1(cre) Ifo/NJU) (Cat.J004781) were kindly provided by Professor Jia-huai Han (Xiamen University). All of the mice were approved by the Animal Care and Use Committee of Xiamen University and maintained with 12-h light/12-h dark cycles at the Laboratory Animal Center in Xiamen University.

Antibody and reagent. Anti-RXRa (D20) (Cat. sc-553,1:1000), anti-RXRa $(\triangle \mathrm{N} 197)$ (Cat. sc-774,1:1000), anti-Myc (9E10) (Cat. Sc-40, 1:1000), anti-TRAF6 $(\mathrm{H}-274)$ (Cat. sc-7221, 1:1000), anti-AKT (Cat. sc-8312, 1:1000), anti- $\beta$-catenin (E5, 1:1000) (Cat. sc-7963), anti-PCNA (FC-261, 1:100 dilution for immunohistochemistry) (Cat. sc7907), and anti-p65 (Cat. sc-109) antibodies were from Santa Cruz Biotechnology (Santa Cruz, CA, USA). Anti-IкBa (Cat. ab32518, 1:1000), anti-pSTAT3 (Y705) (Cat. ab76315, 1:100,000), anti-Ki-67 (Cat.ab15580, 1:200 dilution for immunohistochemistry), and anti-CD68 (Cat. ab31630, 1:400 dilution for immunohistochemistry) antibodies were from Abcam (UK). Anti-Ubiquitin (Cat. 3933S), anti-STAT3 (Cat. 9139S, 1:1000), anti-p-IkBa (Cat. 9246, 1:1000) anti-K63-ubiquitin (D7A11) (Cat.\#5621, 1:1000), anti-TRAF6 (D21G3) (Cat. 8028S, 1:1000), anti-pGSK3 $\beta$ (Ser9) (Cat.\#9336, 1:1000), and anti-pAKT (S473) (Cat. 9271S, 1:1000) antibodies were from Cell Signal Technology (Beverly, MA, USA). Anti-GSK3 $\beta$ (total) (Cat.610202, 1:1000) was from BD biosciences (San Diego, USA). Anti- $\beta$-actin (Cat. A5441, 1:50,000) and anti-FLAG (Cat. F1804, 1:1000) antibodies were from Sigma (St. Louis, MO, USA). DSS (Cat. 0216011080100g) (MW:36000-50000) was from MP Biomedicals. AOM (Cat. A5486), LPS (Cat. L2630), RXRa siRNA (SASI_Hs01_00097639, SASI_Hs01_00097640,SASI_Rn02_00260246), and nonspecific control siRNA were from Sigma. TianScript RT Kit (Cat. KR104-02) was from TianGen Biotechnology (Beijing, China). Mouse IL-6 (Cat. KE10007) and TNFa (Cat. KE10002) enzyme-linked immunosorbent assay kits for IL- 6 and TNFa were from Proteintech (Wuhan, China).

Induction of colitis and CAC. Mice (8-12-week old, male) were injected intraperitoneally with a single dose of AOM $(10 \mathrm{mg} / \mathrm{kg}$; Sigma, \#A5486). After 7 days, $2.5 \%$ DSS (MP Biomedicals, \#0216011080100g) was given in the drinking water for 7 days, followed by 14 days of regular drinking water. The DSS treatment was repeated for two additional cycles, and mice were sacrificed 120 days after the AOM injection. In the K-80003 treatment model, K-80003 (dissolved in dimethyl sulfoxide (DMSO) and diluted with $0.9 \% \mathrm{NaCl}$ containing $5.0 \%(\mathrm{~V} / \mathrm{V})$ Tween-80, $20 \mathrm{mg} / \mathrm{kg}$ ) were administered via oral gavage daily in last 2 weeks. Normal saline with DMSO and 5.0\% Tween-80 was employed as the vehicle control. For shortterm colitis and inflammation studies, mice (8-10-week old) were given $3 \%$ DSS 
for 5 days, followed by regular drinking water for 4 days and sacrificed at the indicated time points. Body weights were recorded daily. Colons were removed from mice, flushed with cold phosphate-buffered saline (PBS), opened longitudinally, fixed as "swiss-rolls" in $4 \%$ formalin solution (Sigma, \#16005-1KG-R), and paraffin embedded. Before fixing the colons, tumor size measurements were performed using a vernier caliper in a blinded fashion.

Clinical tumor samples. Human colorectal cancerous and para-cancerous tissues were obtained from Zhongshan Hospital, Xiamen University, and human colorectal tissues from normal and ulcerative colitis patients were obtained from Soochow University hospital with patient informed consent and approval of the Medical Ethical Committee of Zhongshan Hospital and Soochow University hospital, respectively.

Histopathological analysis and immunohistochemistry. Paraffin-embedded colon sections were stained with H\&E. The extent of inflammation was measured and scored. Paraffin-embedded slides were deparaffinized. Antigen unmasking was carried out by heating in microwave in $10 \mathrm{mM}$ sodium citrate buffer for $5 \mathrm{~min}$ and cooling for $2 \mathrm{~min}$, with repeat of this process five times. Slides were incubated with primary antibodies in PBS. Horseradish peroxidase secondary anti-rat or anti-rabbit antibodies (BIOTnA, cat\#TAHC03D) were added and incubated at room temperature for $1 \mathrm{~h}$. The sections were stained with DAB substrate and counterstained with hematoxylin. 10-15 fields were randomly selected from each section at high magnification, photographed, and analyzed by two pathologists previously uninformed. Histological scores were assigned by experimenters "blinded" to sample identity. Colonic epithelial damage was assigned scores as follows: $0=$ normal; $1=$ hyperproliferation, irregular crypts, and goblet cell loss; $2=$ mild-to-moderate crypt loss (10-50\%); $3=$ severe crypt loss (50-90\%); $4=$ complete crypt loss, surface epithelium intact; $5=$ small-to-medium-sized ulcer $(<10$ crypt widths); $6=$ large ulcer $(\geq 10$ crypt widths).

Real-time polymerase chain reaction. Total RNAs were extracted by Trizol (Invitrogen) and complemental DNA were synthesized using TIANScript RT Kit (Tiangen). Real-time PCR was performed using SYBR Green Master Mix following the manufacturer's instructions (Roche) on ABI Stepone PCR instrument using respective primers (Supplementary Table 1). Expression data were normalized to GAPDH mRNA expression.

Cell culture and transfection. RAW264.7, CV-1 cells, THP-1, and HEK293T were purchased from the American Type Culture Collection (Manassas, VA, USA). CT26.WT and HCT-116 were purchased form Cell Bank in Chinese Academy of Sciences in Shanghai. RAW264.7, CV-1 cells, and HEK293T cells were cultured in Dulbecco's modified Eagle's medium (DMEM) with 10\% fetal bovine serum (FBS); THP-1 cells were maintained in RPMI 1640 containing $10 \%$ fetal bovine serum in a humidified atmosphere containing $5 \% \mathrm{CO}_{2}$ at $37^{\circ} \mathrm{C}$. They were regularly tested for mycoplasma contamination using the MycoFluor ${ }^{\mathrm{TM}}$ Mycoplasma Detection Kit (ThermoFisher Scientific) and always found to be negative. Transient transfection of HEK293T cells were carried out with calcium phosphate method. RAW264.7 cells, THP-1 cells, and CV-1 cells were transfected with Lipofectamin 2000 (Invitrogen). RAW264.7 stable cells lines expressing GFP, GFP-RXR $\alpha$, and GFPtRXRa were selected with $600 \mu \mathrm{g} / \mathrm{ml} \mathrm{G} 418$ for 3-4 weeks.

Isolation and culture of BMDMs. Bone marrow cells were flushed from the femurs and tibias and treated with red blood cell lysis buffer (BD Biosciences, cat555899) to get rid of blood cell. Single-cell suspensions were obtained by forcing through a 40-mm cell strainer and differentiated into macrophages in DMEM supplemented with $10 \% \mathrm{FBS}, 30 \% \mathrm{~L} 929 \mathrm{CM}$, and $1 \%$ penicillin and streptomycin for 6-7days. Primary BMDMs were cultured with DMEM supplemented with $10 \%$ FBS for experiments.

Condition medium and colony-formation assay. BMDMs were cultured with DMEM supplemented with $10 \%$ FBS for $72 \mathrm{~h}$. THP-1 cells were cultured with RPMI1640 without FBS for $48 \mathrm{~h}$. These media were collected, centrifuged with $10,000 \times g$ for $20 \mathrm{~min}$ and stored at $-80^{\circ} \mathrm{C}$. For culturing tumor cells, CM were warmed up at $4{ }^{\circ} \mathrm{C}$ and used to culture tumor cells. In the colony assay, media were used to culture tumor cells for 2 weeks in 6-well plate and colonies were fixed with methylalcohol and stained with Giemsa.

Cell invasion assay. Cell invasion assay was performed with an invasion chamber (BD Biosciences, cat \#354480) pretreated with medium $2 \mathrm{~h}$ for matrix membrane hydration. Tumor cells (HCT116 or MDA-MB-231, $2.5 \times 10^{4}$ per well, $1 \%$ FBS) were seeded in the upper chamber, and the inflammatory cells (THP-1 cell, $1 \times 10^{5}$ per well, $1 \%$ FBS) were loaded into the lower chamber. After $36 \mathrm{~h}$ of incubation at $37^{\circ} \mathrm{C}$ in $5 \% \mathrm{CO}_{2}$, noninvasive cells were removed from the upper surface of the transwell membrane with a cotton swab, and the invaded cells on the lower membrane surface were fixed with paraformaldehyde (PFA) and stained with Gimasa. Randomly selected fields were photographed and the numbers of migrated cells per field were counted. For invasion assay using primary BMDMs and murine CT26 colon cancer cells, CM from BMDMs were placed in the lower chamber and CT26 colon cell were seeded in the upper chamber.

Plasmid constructions. Expression constructs for TRAF6 was kindly provided by Dr. Ashley Mansell (Monash Institute of Medical Research, Australia). TRAF6/ $\triangle \mathrm{TRAF}$ and TRAF6/ $\triangle \mathrm{RING}$ were generated by PCR and inserted into $p C M V-M y c$ vector. Plasmids encoding RXRa, RXRa/1-135, RXRa/1-235, and RXRa/223-462 were constructed using pEGFP-C1 vector.

Immunoprecipitation and ubiquitination assay. Cells were lysed in a buffer containing $2 \mathrm{mmol} / \mathrm{l} \mathrm{Tris-HCl}$ (pH7.4), $10 \mathrm{mmol} / \mathrm{l} \mathrm{EDTA}, 100 \mathrm{mmol} / \mathrm{l} \mathrm{NaCl}$, and $1 \%$ IGEPAL. Whole-cell lysates were subjected to immunoprecipitation with antibodies against RXRa (D20), RXRa ( $\triangle \mathrm{N} 197), \mathrm{TRAF6}$, or c-Myc in protein A/G beads (Santa Cruz Biotechnology). For detection of TRAF6 ubiquitination, $10 \mathrm{mM}$ $\mathrm{N}$-ethylmaleimide was included in the lysis buffer containing a protease inhibitor "cocktail" (Roche).

Immunoblotting (IB). IB was performed as described previously ${ }^{21,25,52}$. Briefly, cell or tissue lysates were electrophoresed by sodium dodecyl sulfatepolyacrylamide gel electrophoresis and transferred to polyvinylidene difluoride membranes. The membranes were blocked with 5\% skimmed milk in TBST (50 $\mathrm{mM}$ Tris- $\mathrm{HCl}(\mathrm{pH} 7.4), 150 \mathrm{mM} \mathrm{NaCl}$, and $0.1 \%$ Tween20) for $1 \mathrm{~h}$, then incubated with primary antibodies and secondary antibodies and detected using enhanced chemiluminescence. The original immunoblots from the main figures are shown in Supplementary Figure 8.

Confocal microscopy. Cells seeded on glass slides were washed with PBS and fixed in 4\% PFA for 15 min. Fixed cells were permeabilized with PBS containing $0.1 \%$ Triton X-100 for $15 \mathrm{~min}$ and blocked with $1 \mathrm{mg} / \mathrm{ml}$ bovine serum albumin in PBS for $30 \mathrm{~min}$ at room temperature. Primary antibodies were incubated for 3 $\mathrm{h}$ at room temperature or overnight at $4{ }^{\circ} \mathrm{C}$ and secondary antibodies were incubated at room temperature for $1 \mathrm{~h}$. DAPI was dyed for visualizing nuclei. The images were taken under an LSM-510 confocal laser scanning microscope system (Carl Zeiss).

Luciferease report assay. RAW264.7 and THP-1 cells were transfected with plasmids combined with pNF-kB-Luc for NF-kB reporter. After transfection for 24 $\mathrm{h}$ ( $48 \mathrm{~h}$ for siRNA), cells were lysed by reporter lysis buffer. Firefly and Renilla luciferase activities were analyzed with the Dual-Luciferase Reporter Assay system (Promega). Transfection and expression efficiency were normalized to Renilla luciferase activity

Statistical analysis. Data are presented as mean \pm standard error of the mean (SEM) or \pm standard deviation (SD), and statistical significance are reported in the figure legends. Statistics was analyzed using Student's $t$ test, chi-square test, or analysis of variance (ANOVA) analysis (one-way ANOVA for comparisons between groups, two-way ANOVA for comparisons the magnitude of changes between different groups from different cell lines). $P<0.05$ was considered statistically significant $\left({ }^{*}\right), P<0.01$ as highly significant $\left(^{* *}\right), P<0.001$ as extremely significant $\left({ }^{* * *}\right)$, and $n s$ as not significant.

Reporting summary. Further information on experimental design is available in the Nature Research Reporting Summary linked to this article.

\section{Data availability}

Supporting data of this study are available from the corresponding author on reasonable request. The source underlying Figs. $2 \mathrm{a}, \mathrm{b}, \mathrm{f}, 4 \mathrm{f}, 5 \mathrm{c}, \mathrm{f}, \mathrm{h}, 6 \mathrm{a}, \mathrm{e}-\mathrm{i}, 7 \mathrm{a}, \mathrm{b}, \mathrm{g}$ and Supplementary Figs. 1c, 4c, 4d, 5a, 5c, 6a, 6c-f, 7e, 7g, 7j are provided as a Supplementary Data 1 . A reporting summary for this article is available as a Supplementary Information file.

Received: 30 July 2018 Accepted: 18 February 2019 Published online: 01 April 2019

\section{References}

1. Altucci, L., Leibowitz, M. D., Ogilvie, K. M., de Lera, A. R. \& Gronemeyer, H. RAR and RXR modulation in cancer and metabolic disease. Nat. Rev. Drug Discov. 6, 793-810 (2007).

2. Evans, R. M. \& Mangelsdorf, D. J. Nuclear receptors, RXR, and the Big Bang. Cell 157, 255-266 (2014).

3. Szanto, A. et al. Retinoid X receptors: X-ploring their (patho)physiological functions. Cell Death Differ. 11, S126-S143 (2004). 
4. Huang, J. et al. Prostatic intraepithelial neoplasia in mice with conditional disruption of the retinoid $\mathrm{X}$ receptor alpha allele in the prostate epithelium. Cancer Res. 62, 4812-4819 (2002).

5. Li, M. et al. Skin abnormalities generated by temporally controlled RXRalpha mutations in mouse epidermis. Nature 407, 633-636 (2000).

6. Ocadiz-Delgado, R., Castaneda-Saucedo, E., Indra, A. K., Hernandez-Pando, R. \& Gariglio, P. Impaired cervical homeostasis upon selective ablation of RXRalpha in epithelial cells. Genesis 46, 19-28 (2008).

7. Su, Y. et al. Recent progress in the design and discovery of RXR modulators targeting alternate binding sites of the receptor. Curr. Top. Med. Chem. 17, 663-675 (2017).

8. Zhang, X., Zhou, H. \& Su, Y. Targeting truncated RXRalpha for cancer therapy. Acta Biochim. Biophys. Sin. (Shanghai). 48, 49-59 (2016).

9. Zhang, X. K. et al. Regulation of the nongenomic actions of retinoid X receptor-alpha by targeting the coregulator-binding sites. Acta Pharmacol. Sin. 36, 102-112 (2015).

10. Zeisig, B. B. et al. Recruitment of RXR by homotetrameric RARalpha fusion proteins is essential for transformation. Cancer Cell 12, 36-51 (2007).

11. Zhu, J. et al. RXR is an essential component of the oncogenic PML/RARA complex in vivo. Cancer Cell 12, 23-35 (2007).

12. Casas, F. et al. Endocrine regulation of mitochondrial activity: involvement of truncated RXRalpha and c-Erb Aalphal proteins. FASEB J. 17, 426-436 (2003).

13. Matsushima-Nishiwaki, R., Shidoji, Y., Nishiwaki, S., Moriwaki, H. \& Muto, Y. Limited degradation of retinoid X receptor by calpain. Biochem. Biophys. Res. Commun. 225, 946-951 (1996).

14. Matsushima-Nishiwaki, R. et al. Aberrant metabolism of retinoid X receptor proteins in human hepatocellular carcinoma. Mol. Cell. Endocrinol. 121, 179-190 (1996).

15. Nagaya, T. et al. Intracellular proteolytic cleavage of 9-cis-retinoic acid receptor alpha by cathepsin L-type protease is a potential mechanism for modulating thyroid hormone action. J. Biol. Chem. 273, 33166-33173 (1998).

16. Prufer, K., Schroder, C., Hegyi, K. \& Barsony, J. Degradation of RXRs influences sensitivity of rat osteosarcoma cells to the antiproliferative effects of calcitriol. Mol. Endocrinol. 16, 961-976 (2002).

17. Nomura, Y., Nagaya, T., Yamaguchi, S., Katunuma, N. \& Seo, H. Cleavage of RXRalpha by a lysosomal enzyme, cathepsin L-type protease. Biochem. Biophys. Res. Commun. 254, 388-394 (1999).

18. Takiyama, Y. et al. Decreased expression of retinoid X receptor isoforms in human thyroid carcinomas. J. Clin. Endocrinol. Metab. 89, 5851-5861 (2004).

19. Zhong, C., Yang, S., Huang, J., Cohen, M. B. \& Roy-Burman, P. Aberration in the expression of the retinoid receptor, RXRalpha, in prostate cancer. Cancer Biol. Ther. 2, 179-184 (2003).

20. Chen, F. et al. Identification of a new RXRalpha antagonist targeting the coregulator-binding site. ACS Med. Chem. Lett. 5, 736-741 (2014).

21. Chen, L. et al. Sulindac-derived RXRalpha modulators inhibit cancer cell growth by binding to a novel site. Chem. Biol. 21, 596-607 (2014).

22. Gao, W. et al. Regulation of proteolytic cleavage of retinoid X receptor-alpha by GSK-3beta. Carcinogenesis 34, 1208-1215 (2013).

23. Wang, G. H. et al. Targeting truncated retinoid X receptor-alpha by CF31 induces TNF-alpha-dependent apoptosis. Cancer Res. 73, 307-318 (2013).

24. Wang, Z. G. et al. Synthesis and SAR study of modulators inhibiting tRXRalpha-dependent AKT activation. Eur. J. Med. Chem. 62, 632-648 (2013).

25. Zhou, H. et al. NSAID sulindac and its analog bind RXRalpha and inhibit RXRalpha-dependent AKT signaling. Cancer Cell 17, 560-573 (2010).

26. Zeng, Z. et al. Nitrostyrene derivatives act as RXRalpha ligands to inhibit TNFalpha activation of NF-kappaB. Cancer Res. 75, 2049-2060 (2015).

27. Dawson, M. I. \& Zhang, X.-K. Discovery and design of retinoic acid receptor and retinoid X receptor class- and subtype-selective synthetic analogs of alltrans-retinoic acid and 9-cis-retinoic acid. Curr. Med. Chem. 9, 623-637 (2002).

28. de Lera, A. R., Bourguet, W., Altucci, L. \& Gronemeyer, H. Design of selective nuclear receptor modulators: RAR and RXR as a case study. Nat. Rev. Drug Discov. 6, 811-820 (2007).

29. Liby, K. T., Yore, M. M. \& Sporn, M. B. Triterpenoids and rexinoids as multifunctional agents for the prevention and treatment of cancer. Nat. Rev. Cancer 7, 357-369 (2007).

30. Uray, I. P., Dmitrovsky, E. \& Brown, P. H. Retinoids and rexinoids in cancer prevention: from laboratory to clinic. Semin. Oncol. 43, 49-64 (2016).

31. DiDonato, J. A., Mercurio, F. \& Karin, M. NF-kappaB and the link between inflammation and cancer. Immunol. Rev. 246, 379-400 (2012).

32. Mantovani, A., Allavena, P., Sica, A. \& Balkwill, F. Cancer-related inflammation. Nature 454, 436-444 (2008)

33. Roszer, T., Menendez-Gutierrez, M. P., Cedenilla, M. \& Ricote, M. Retinoid X receptors in macrophage biology. Trends Endocrinol. Metab. 24, 460-468 (2013).
34. Ma, F. et al. Retinoid X receptor alpha attenuates host antiviral response by suppressing type I interferon. Nat. Commun. 5, 5494 (2014).

35. Nunez, V. et al. Retinoid X receptor alpha controls innate inflammatory responses through the up-regulation of chemokine expression. Proc. Natl Acad. Sci. USA 107, 10626-10631 (2010).

36. Lehman, A. M. et al. Activation of the retinoid X receptor modulates angiotensin II-induced smooth muscle gene expression and inflammation in vascular smooth muscle cells. Mol. Pharmacol. 86, 570-579 (2014).

37. Tunctan, B. et al. Bexarotene, a selective RXRalpha agonist, reverses hypotension associated with inflammation and tissue injury in a rat model of septic shock. Inflammation 41, 337-355 (2018).

38. Janakiram, N. B. et al. Chemopreventive effects of RXR-selective rexinoid bexarotene on intestinal neoplasia of $\mathrm{Apc}(\mathrm{Min} /+)$ mice. Neoplasia 14, 159-168 (2012).

39. Grenningloh, R. et al. Cutting edge: Inhibition of the retinoid $\mathrm{X}$ receptor (RXR) blocks $\mathrm{T}$ helper 2 differentiation and prevents allergic lung inflammation. J. Immunol. 176, 5161-5166 (2006).

40. Cao, M. et al. The rexinoids LG100268 and LG101506 inhibit inflammation and suppress lung carcinogenesis in A/J mice. Cancer Prev. Res. 9, 105-114 (2016).

41. de Urquiza, A. M. et al. Docosahexaenoic acid, a ligand for the retinoid X receptor in mouse brain. Science 290, 2140-2144 (2000).

42. Kolluri, S. K. et al. The R-enantiomer of the nonsteroidal antiinflammatory drug etodolac binds retinoid $\mathrm{X}$ receptor and induces tumor-selective apoptosis. Proc. Natl Acad. Sci. USA 102, 2525-2530 (2005).

43. Rochette-Egly, C. Retinoic acid signaling and mouse embryonic stem cell differentiation: Cross talk between genomic and non-genomic effects of RA. Biochim. Biophys. Acta 66-75, 2015 (1851).

44. $\mathrm{Li}, \mathrm{H}$. et al. Cytochrome $\mathrm{c}$ release and apoptosis induced by mitochondrial targeting of nuclear orphan receptor TR3 [see comments] [comment]. Science 289, 1159-1164 (2000).

45. Lin, B. et al. Conversion of Bcl-2 from protector to killer by interaction with nuclear orphan receptor Nur77/TR3. Cell 116, 527-540 (2004).

46. $\mathrm{Hu}, \mathrm{M}$. et al. Celastrol-induced Nur77 interaction with TRAF2 alleviates inflammation by promoting mitochondrial ubiquitination and autophagy. Mol. Cell 66, 141-153 (2017).

47. Kousteni, S. et al. Nongenotropic, sex-nonspecific signaling through the estrogen or androgen receptors: dissociation from transcriptional activity. Cell 104, 719-730 (2001).

48. Simoncini, T. et al. Interaction of oestrogen receptor with the regulatory subunit of phosphatidylinositol-3-OH kinase. Nature 407, 538-541 (2000).

49. Ghose, R., Zimmerman, T. L., Thevananther, S. \& Karpen, S. J. Endotoxin leads to rapid subcellular re-localization of hepatic RXRalpha: a novel mechanism for reduced hepatic gene expression in inflammation. Nucl. Recept. 2, 4 (2004).

50. Zimmerman, T. L., Thevananther, S., Ghose, R., Burns, A. R. \& Karpen, S. J. Nuclear export of retinoid X receptor alpha in response to interleukin-1betamediated cell signaling: roles for JNK and SER260. J. Biol. Chem. 281, 15434-15440 (2006)

51. Davies, N. M. \& Watson, M. S. Clinical pharmacokinetics of sulindac. A dynamic old drug. Clin. Pharmacokinet. 32, 437-459 (1997).

52. Chen, L. et al. Modulation of nongenomic activation of PI3K signalling by tetramerization of N-terminally-cleaved RXRalpha. Nat. Commun. 8, 16066 (2017).

53. Giardiello, F. M. et al. Treatment of colonic and rectal adenomas with sulindac in familial adenomatous polyposis. N. Engl. J. Med. 328, 1313-1316 (1993).

54. Samadder, N. J. et al. Association of sulindac and erlotinib vs placebo with colorectal neoplasia in familial adenomatous polyposis: secondary analysis of a randomized clinical trial. JAMA Oncol. 4, 671-677 (2018).

55. Cuzick, J. et al. Aspirin and non-steroidal anti-inflammatory drugs for cancer prevention: an international consensus statement. Lancet Oncol. 10, 501-507 (2009).

56. Yamazaki, K. et al. Synergistic effects of RXR alpha and PPAR gamma ligands to inhibit growth in human colon cancer cells-phosphorylated RXR alpha is a critical target for colon cancer management. Gut 56, 1557-1563 (2007).

57. Neufert, C., Becker, C. \& Neurath, M. F. An inducible mouse model of colon carcinogenesis for the analysis of sporadic and inflammation-driven tumor progression. Nat. Protoc. 2, 1998-2004 (2007).

58. Bissahoyo, A. et al. Azoxymethane is a genetic background-dependent colorectal tumor initiator and promoter in mice: effects of dose, route, and diet. Toxicol. Sci. 88, 340-345 (2005).

59. Xiao, J. H. et al. Adenomatous polyposis coli (APC)-independent regulation of beta-catenin degradation via a retinoid X receptor-mediated pathway. J. Biol. Chem. 278, 29954-29962 (2003).

60. Rayasam, G. V., Tulasi, V. K., Sodhi, R., Davis, J. A. \& Ray, A. Glycogen synthase kinase 3: more than a namesake. Br. J. Pharmacol. 156, 885-898 (2009). 
61. Bollrath, J. et al. gp130-mediated Stat3 activation in enterocytes regulates cell survival and cell-cycle progression during colitis-associated tumorigenesis. Cancer Cell 15, 91-102 (2009).

62. Grivennikov, S. et al. IL-6 and Stat 3 are required for survival of intestinal epithelial cells and development of colitis-associated cancer. Cancer Cell 15 103-113 (2009).

63. Clausen, B. E., Burkhardt, C., Reith, W., Renkawitz, R. \& Forster, I. Conditional gene targeting in macrophages and granulocytes using LysMcre mice. Transgenic Res. 8, 265-277 (1999).

64. Chen, Z. J. Ubiquitination in signaling to and activation of IKK. Immunol. Rev. 246, 95-106 (2012)

65. Walsh, M. C., Lee, J. \& Choi, Y. Tumor necrosis factor receptor- associated factor 6 (TRAF6) regulation of development, function, and homeostasis of the immune system. Immunol. Rev. 266, 72-92 (2015).

66. Sun, H. et al. TRAF6 is upregulated in colon cancer and promotes proliferation of colon cancer cells. Int. J. Biochem. Cell Biol. 53, 195-201 (2014).

67. Zhang, T., Wang, H. \& Han, L. Expression and clinical significance of tumor necrosis factor receptor-associated factor 6 in patients with colon cancer. Iran. Red Crescent Med. J. 18, e23931 (2016).

68. $\mathrm{Wu}, \mathrm{H}$. et al. NUR77 exerts a protective effect against inflammatory bowel disease by negatively regulating the TRAF6/TLR-IL-1R signalling axis. J. Pathol. 238, 457-469 (2016).

69. Starczynowski, D. T. et al. TRAF6 is an amplified oncogene bridging the RAS and NF-kappaB pathways in human lung cancer. J. Clin. Invest. 121, 4095-4105 (2011).

70. Borer, J. S. \& Simon, L. S. Cardiovascular and gastrointestinal effects of COX-2 inhibitors and NSAIDs: achieving a balance. Arthritis Res. Ther. 7, S14-S22 (2005).

\section{Acknowledgements}

We thank Professor Jianming Li from Soochow University for technical assistance. This work was partially supported by grants from the Natural Science Foundation of China (81672749, U1405229, 81741171, 91429306), National Institute of Health (RO1 CA198982), Regional Demonstration of Marine Economy Innovative Development Project (16PYY007SF17), Fujian Provincial Science \& Technology Department (2017YZ0002), and the National Institute of Health (R01CA198982).

\section{Author contributions}

X.Y. generated transgenic mice; X.Y and H.W. conducted cellular and molecular experiments; X.Y., L.S., Y.-x.L., F.Y. and M.W. constructed animal and compound evaluation experiments; H.Z., Y.S. and X.-k.Z. analyzed data; X.Y., Y.S. and X.-k.Z. wrote the manuscript.

\section{Additional information}

Supplementary Information accompanies this paper at https://doi.org/10.1038/s41467019-09375-8.

Competing interests: The authors declare no competing interests.

Reprints and permission information is available online at http://npg.nature.com/ reprintsandpermissions/

Journal peer review information: Nature Communications thanks John Welch and the other anonymous reviewer(s) for their contribution to the peer review of this work.

Publisher's note: Springer Nature remains neutral with regard to jurisdictional claims in published maps and institutional affiliations.

cc) (i) Open Access This article is licensed under a Creative Commons Attribution 4.0 International License, which permits use, sharing, adaptation, distribution and reproduction in any medium or format, as long as you give appropriate credit to the original author(s) and the source, provide a link to the Creative Commons license, and indicate if changes were made. The images or other third party material in this article are included in the article's Creative Commons license, unless indicated otherwise in a credit line to the material. If material is not included in the article's Creative Commons license and your intended use is not permitted by statutory regulation or exceeds the permitted use, you will need to obtain permission directly from the copyright holder. To view a copy of this license, visit http://creativecommons.org/ licenses/by/4.0/.

(C) The Author(s) 2019 